\title{
The bacterial magnesium transporter MgtA reveals highly selective interaction with specific cardiolipin species
}

Julia Weikum ${ }^{1,2}$, Jeroen van Dyck ${ }^{3}$, Saranya Subramani ${ }^{1}$, David P. Klebl ${ }^{4}$, Merete Storflor ${ }^{5}$, Stephen P. Muench ${ }^{4}$, Sören Abel ${ }^{5}$, Frank Sobott *6, J. Preben Morth *1,2,7

${ }^{1}$ Membrane Transport Group, Centre for Molecular Medicine Norway (NCMM), Nordic EMBL Partnership, University of Oslo, P.O. Box 1137 Blindern, 0318 Oslo, Norway.

${ }^{2}$ Enzyme and Protein Chemistry, Section for Protein Chemistry and Enzyme Technology, Department of Biotechnology and Biomedicine, Technical University of Denmark, Søltofts Plads, 2800, Kgs. Lyngby, Denmark.

${ }^{3}$ Department of Chemistry, University of Antwerp, Campus Groenenborger, Groenenborgerlaan 171, G.V. 418, 2020 Antwerpen, Belgium.

4 School of Biomedical Sciences \& The Astbury Centre for Structural Molecular Biology, University of Leeds, Woodhouse Lane, Leeds LS2 9JT, United Kingdom.

5 The Abel Lab, Department of Pharmacy, UiT-The Arctic University of Norway, 9037 Tromsø, Norway.

6 School of Molecular and Cellular Biology \& The Astbury Centre for Structural Molecular Biology, University of Leeds, Woodhouse Lane, Leeds LS2 9JT, United Kingdom.

${ }^{7}$ Institute for Experimental Medical Research (IEMR), Oslo University Hospital, Ullevål PB 4956 Nydalen, NO-0424 Oslo, Norway.

* correspondence should be addressed to f.sobott@leeds.ac.uk or premo@dtu.dk

\section{Abstract}

A significant challenge today within protein lipidology is to understand the relationship between cell structure, lipid membrane integrity, ion homeostasis and the embedded membrane proteins. 6 The bacterial magnesium transporter A (MgtA) is a specialized P-type ATPase important for $\mathrm{Mg}^{2+}$ import into the cytoplasm; disrupted magnesium homeostasis is linked to intrinsic ribosome instability and nitro-oxidative stress in Salmonella strains. MgtA's function is highly dependent on anionic lipids, particularly cardiolipin, and further co-localization of cardiolipin with MgtA at 
the $E$. coli cell poles has been revealed. Here, we show that MgtA has functional specificity for cardiolipin 18:1, but it reaches maximum activity only in combination with cardiolipin 16:0, equivalent to the major components of native cardiolipin found in E. coli membranes. This is the first time it has been shown experimentally that two different lipid species from the same class, individually promoting low activity, can enhance activity in combination. Native mass spectrometry verifies the presence of two binding sites for cardiolipin and kinetic studies reveal that a cooperative relationship likely exists between the two different cardiolipin variants (cardiolipin 16:0 and cardiolipin 18:1). This is the first experimental evidence of cooperative effects between lipids of the same class, with only minor variations in their acyl chain composition, acting on a membrane protein. In summary, our results reveal that MgtA exhibits a highly complex interaction with one cardiolipin 18:1 and one cardiolipin 16:0, affecting protein activity and stability, and contributing to our understanding of the particular interactions between lipid environment and membrane proteins. Further, a better understanding of $\mathrm{Mg}^{2+}$ homeostasis in bacteria, due to its role as a virulence regulator, will provide further insights into the regulation and mechanism of bacterial infections.

\section{Introduction}

Biological membranes function as a dynamic interplay between proteins and lipids. The plasma membrane contains a specific lipid composition which can differ locally, and these regions can accommodate functional specialization based on non-random lipid-lipid and lipid-protein interactions (1). The lipid ensemble reveals a high degree of molecular variation in both the head group structure which presents various functional groups at the membrane surface, and in the acyl chains with their variable length and degree of saturation (2), located within the transmembrane segment. This combinatorial space opens the possibility of an additional regulatory function by lipids of the same type which differ only marginally in their acyl chain composition, but have unique structural and functional properties (3). Significant progress has been made in elucidating the intricate and complex interplay between membrane proteins and lipids from experimental studies $(4,5)$. For several P-type ATPase family members, a role of specific lipid interactions has been elucidated, specifically the plasma membrane $\mathrm{Ca}^{2+}$-ATPase (PMCA) and dimyristoyl phosphatidylcholine-dependent activity variation between PMCA2 and PMCA4 (6), and lipid- 
protein stoichiometric variations dependent on the functional state of the PMCA (6). The prominent $\mathrm{Na}^{+} / \mathrm{K}^{+}$-ATPase is strongly stimulated and inhibited by lipid-protein interactions (7); of particular importance is the modulatory effect of cholesterol (8) and the stimulatory effect of polyunsaturated lipids such a phosphatidylethanolamine (PE) and phosphatidylserine (PS) (9). The stimulus mainly accelerates the E1P-E2P conformational transition and is mediated by two independent lipid binding sites, with a PS lipid bound between $\alpha$ TM8-10 and the FXYD transmembrane helix, and a PE bound between $\alpha \mathrm{M} 2,4,6$, and 9 (10).

Membrane proteins from bacterial species have also been investigated and while many, however not all, show selective lipid binding (4), their function role often remains poorly understood. Typically, structural studies have revealed the molecular basis of various lipid binding sites in membrane proteins. Specific binding pockets for lipids are referred to as «non-annular lipid sites», characterized through longer lipid residence times (11). They are stabilized through polar interactions between head groups and charged amino acids at the water-lipid interface or hydrophobic interactions between acyl chains and hydrophobic residues in the transmembrane segments (11). Interestingly, the lipid composition of the membrane, which determines the bulk properties of the lipid bilayer, such as membrane fluidity and bilayer thickness, also influences membrane protein function, as shown for certain P-type ATPases (12). Lipids that associate weakly with transmembrane segments exhibit faster lipid exchange rates. They have been termed «annular lipids/annular lipid shell», as they often form a ring-like structure around the membraneembedded protein section (11). This study mainly focuses on non-annular, i.e. tightly bound/structural lipid interactions between MgtA and specific cardiolipin species. The magnesium transporter A (MgtA) is a primary active $\mathrm{Mg}^{2+}$ transporter, transporting $\mathrm{Mg}^{2+}$ from the periplasm to the cytoplasm with orthology widely distributed throughout eubacteria, fungi and plants (13, 14). The transporter ensures $\mathrm{Mg}^{2+}$ homeostasis which is essential for stabilising membranes and ribosomes, ensuring correct folding of oligonucleotides, and functions as a co-factor for enzymatic reactions. Additionally, $\mathrm{Mg}^{2+}$ has been revealed as a bacterial virulence regulator (15) and can also protect against hostile environments, such as withstanding nitro-oxidative stress in professional phagocytes during infection (16). Furthermore, a recently discovered role for MgtA and especially its homologue MgtB suggests that the transporters promote survival in macrophages during infections, by preventing $\mathrm{Mg}^{2+}$ deprivation (17). 
MgtA is a P-type ATPase transporter which utilizes energy from the hydrolysis of adenosine triphosphate (ATP) for $\mathrm{Mg}^{2+}$ transport $(15,18)$ and exhibits a classical P-type ATPase structural composition with three cytoplasmic domains, termed nucleotide-binding $(\mathrm{N})$, phosphorylation $(\mathrm{P})$ and actuator (A) domain, and a transmembrane core with ten transmembrane helices $(19,20)$. The expression of MgtA is tightly regulated by the two-component system $\mathrm{PhoP} / \mathrm{PhoQ}$, which upregulates MgtA levels under low extracellular $\mathrm{Mg}^{2+}$ concentrations, low $\mathrm{pH}$ and in the presence of antibacterial peptides (15). The PhoP/PhoQ-induced sigma factor RpoS accumulates as a response to low cytoplasmic $\mathrm{Mg}^{2+}$ levels in Salmonella, tied to the presence of functional $\mathrm{Mg}^{2+}$ homeostasis mediated via MgtA and MgtB in Salmonella and other pathogens (21-23). Additional regulation of MgtA expression is present through the $m g t A$ mRNA leader region, $m g t L$, which senses intracellular $\mathrm{Mg}^{2+}$ levels and only promotes MgtA translation under low intracellular $\mathrm{Mg}^{2+}$ concentrations (24-26). The enzymatic activity of MgtA is also under tight regulation through substrate inhibition by $\mathrm{Mg}^{2+}$ free, which allows MgtA activation only in the concentration range of $10 \mu \mathrm{M}$ to $1 \mathrm{mM} \mathrm{Mg}{ }^{2+}$ free (14). $\mathrm{Mg}^{2+}$ free describes $\mathrm{Mg}^{2+}$ ions that are not coordinated by ATP but are available for transport by MgtA. However, the mechanism of MgtA inhibition by $\mathrm{Mg}^{2+}$ free remains unclear. While most of our understanding of bacterial $\mathrm{Mg}^{2+}$ homeostasis derives from Salmonella, considerable similarities between E. coli, which only has MgtA and not its homologue MgtB, have been described (27).

MgtA is located in the inner membrane of E. coli, which contains only approximately $\sim 5 \%$ cardiolipin (CL), with the remainder being $\sim 75 \%$ phosphatidylethanolamine (PE) and $\sim 20 \%$ phosphatidylglycerol (PG) (28). Previously, we have shown that CL performs an essential function for MgtA activation, and that it co-localizes at the inner membrane of E. coli poles and septal regions (14). Cardiolipin has four acyl chains in comparison to classical glycerophospholipids which only have two. In contrast to eukaryotes, which often have only one or two major cardiolipins (29), E. coli contains a large variety of CL species with variable hydrophobic tails with palmitic acid (16:0), palmitoleic acid (16:1) and oleic acid (18:1) as the most abundant acyl chains (30). It has been proposed previously that the anionic head group of cardiolipin is most critical for MgtA ATPase activity, while the acyl chain composition of the lipid only contributes marginally to the enzyme activation (14). Therefore, a function of cardiolipin as a $\mathrm{Mg}^{2+}$ or $\mathrm{H}^{+}$ chaperone or reservoir for MgtA-mediated $\mathrm{Mg}^{2+}$-transport was hypothesized (14). The data presented here however indicates a more complex interaction between MgtA and cardiolipin. In 
121 this study, we show the specific interaction of MgtA with selected cardiolipin species. Enzyme

122 kinetics studies and native mass spectrometry determined that these lipid-protein interactions are

123 likely mediated through site-specific interactions and biophysical properties of the lipid bilayer

124 environment. We reveal high selectivity of MgtA for CL species 18:1 and CL 16:0, and

125

126

127

128

129

130 cooperativity of binding of these two CL molecules to MgtA. Additionally, we show thermal stabilization of MgtA by CL 18:1 through nano differential scanning fluorimetry, while no effect on MgtA stabilization by CL 16:0 was detected. These results highlight the complexity of lipidmembrane protein interactions, with highly selective roles found even for closely related lipid species varying only slightly in acyl chain structure. The implications of this exquisite fine tuning of membrane protein function might indeed be suggestive of a much greater role of lipid composition than previously anticipated.

\section{Material and Methods}

Lipids were purchased from Avanti Polar Lipids and solubilized in chloroform. E. coli cardiolipin (cat no. 841199), cardiolipin 18:1 (cat no. 710335) and cardiolipin 16:0 (cat no. 710333) were purchased as stock solutions of $10 \mathrm{mg} / \mathrm{ml}$. Cardiolipin 14:1 (cat no. 710337) and cardiolipin 16:1 (cat no. 710339) were obtained as stock solutions of $5 \mathrm{mg} / \mathrm{ml}$. Total E. coli lipid extract (cat no. 100500) and 1-palmitoyl-2-oleoyl-sn-glycero-3-phosphoethanolamine (POPE) (cat no. 850757) were purchased as $25 \mathrm{mg} / \mathrm{ml}$ stock solution. Detergents were obtained from either Chemical Point UG, Deisenhofen, Germany for n-dodecyl- $\beta$-D-maltopyranoside (DDM) or from Nikko Chemicals for octaethylene glycol monododecyl ether $\left(\mathrm{C}_{12} \mathrm{E}_{8}\right)$. All other chemicals were purchased as grade BioUltra from Sigma-Aldrich.

\section{Heterologous expression and purification of MgtA}

Cloning of E. coli MgtA into pETM11 vector (EMBL), heterologous expression in E. coli C43(DE3) and purification were performed as described in Subramani et al. (2016) (14, 31). In brief, isolated E coli membranes with overexpressed MgtA were solubilized in 1\% $\beta$-dodecyl maltoside ( $\beta$-DDM) prior to Histrap HP (GE Healthcare) purification equilibrated in $(25 \mathrm{mM}$ HEPES pH 7.0, 100 mM K $2 \mathrm{SO}_{4}, 5 \%$ glycerol, 1 mM PMSF, $5 \mathrm{mM} \beta$-mercaptoethanol, 3x CMC 
150

151

152

153

154

155

156

157

158

159

160

161

162

163

164

165

166

167

168

169

170

171

172

173

$\beta$-DDM) and eluted in (25 mM HEPES pH 7.0, $100 \mathrm{mM} \mathrm{K}_{2} \mathrm{SO}_{4}, 5 \%$ glycerol, $1 \mathrm{mM}$ PMSF, $5 \mathrm{mM}$ $\beta$-mercaptoethanol, $300 \mathrm{mM}$ imidazole $\mathrm{pH}$ 7.6). The elution was followed by size exclusion chromatography, using a HiLoad 16/600 Superdex 200 pg column equilibrated with $1.5 \mathrm{CV}$ of buffer E buffer E (25 mM HEPES, pH 7.0, $100 \mathrm{mM} \mathrm{K}_{2} \mathrm{SO}_{4}, 5 \%$ glycerol, $1 \mathrm{mM}$ dithiothreitol, 3x CMC $\beta$-DDM).

\section{ATPase enzymatic assay}

Lipid stocks for ATPase enzymatic assays were prepared by tempering indicated lipids, dissolved in chloroform, to room temperature, or heating them to $40{ }^{\circ} \mathrm{C}$ until completely dissolved. The lipids were dried under an argon stream with vigorous shaking. The dried lipid film was resuspended in Milli-Q water and $20 \mathrm{mM} \mathrm{C}_{12} \mathrm{E}_{8}$ with vigorous shaking for at least 1 hour at $\mathrm{RT}$ and sonication intervals of $5-10 \mathrm{~min}$ in between. A final lipid concentration of $6.12 \mathrm{mM}$ or $12.24 \mathrm{mM}$ for lipid profiles or a final concentration of $12 \mathrm{mM}$ for $\mathrm{Mg}^{2+}$ profiles, respectively, was obtained. Fresh lipid stocks were prepared prior to enzymatic assays. For enzymatic assays in the presence of increasing lipid concentrations (lipid profiles), dilutions to indicated concentrations were prepared in Milli-Q water with $20 \mathrm{mM} \mathrm{C}_{12} \mathrm{E}_{8}$. The same volume $(1 \mu \mathrm{L})$ of lipid dilutions was added to the reaction volume to keep detergent levels constant (final concentration: $3 \mathrm{x}$ CMC $\mathrm{C}_{12} \mathrm{E}_{8}$ ), independent of the lipid concentration.

ATPase enzymatic assays measuring ATP hydrolysis by detecting released inorganic phosphate were performed according to the protocol described earlier (14). The online web service MAXC (maxchelator.stanford.edu) was used to calculate $\mathrm{Mg}^{2+}$ free levels in the presence of given concentrations of ATP at $\mathrm{pH} 7.0$ at $37{ }^{\circ} \mathrm{C}$. Apparent $\mathrm{V}_{\max }$ and $\mathrm{K}_{\mathrm{m}}$ were calculated by fitting the curves (specific activity) as a function of $\mathrm{Mg}^{2+}$ free using a modified uncompetitive substrate inhibition model (equation 1)

$$
Y=\frac{V_{\max } X}{K_{m}+X\left(1+\frac{X^{n}}{K_{i}}\right)}
$$


175

176

177

178

179

\section{0}

181

182

183

184

185

186

187

188

189

190

191

192

193

194

195

196

197

198

199

200

201

202

with $\mathrm{X}$ referring to the substrate concentration, $\mathrm{Y}$ to the enzyme activity and $\mathrm{n}$ to the number of uncompetitive inhibitor sites, provided by GraphPad Prism8 (www.graphpad.com). $\mathrm{k}_{\text {cat }}$ was estimated using the formula $\mathrm{k}_{\mathrm{cat}}=\mathrm{V}_{\max } /[\mathrm{MgtA}]$.

The Hill coefficient for CL binding was obtained by fitting the curves using the allosteric sigmoidal equation 2

$$
Y=\frac{V_{\max } X^{h}}{K_{\text {half }}^{h}+X^{h}}
$$

provided by GraphPad Prism8 with X referring to the substrate concentration, $\mathrm{Y}$ to the enzyme activity, $\mathrm{K}_{\text {half }}$ to the substrate concentration that produces half-maximal enzyme velocity and $\mathrm{h}$ to the Hill slope.

\section{Native Mass Spectrometry}

Native mass spectrometry (native MS) was performed on purified MgtA protein, subsequently incubated with lipids depending on the experiment. For lipid selectivity of MgtA, total E. coli lipid extract (consisting of $67 \%$ PE, $23 \%$ PG and $10 \%$ CL) was used, while selected lipids, POPE and CL, were included for competition assays. Chloroform, in which lipids were dissolved, was removed under an argon stream until the lipid film was completely dried. The lipid film was resuspended in buffer (25 mM HEPES, pH 7.0; $200 \mathrm{mM} \mathrm{KCl} ; 5 \%$ Glycerol) and 6x CMC (1.02 mM) DDM for a final concentration of $1.47 \mathrm{mg} / \mathrm{ml}$ for total E. coli lipid (for lipid selectivity) or $400 \mu \mathrm{M}$ for CL and $2.4 \mathrm{mM}$ for POPE (for competition assays). For the competition assays, CL and POPE were mixed in the indicated ratio. MgtA was diluted in the buffer to a final concentration of $20 \mu \mathrm{M}$. $25 \mathrm{ul} \mathrm{MgtA}(20 \mu \mathrm{M})$ were mixed with $25 \mu$ lipid solution and incubated for $16 \mathrm{~h}$ at $4{ }^{\circ} \mathrm{C}$ in a rotator $(5 \mathrm{rpm})$.

MgtA protein samples had to be desalted prior to the native MS measurement. This was accomplished by washing the sample several times in a $100 \mathrm{kDa}$ cut-off concentrator column, with the cut-off small enough to remove most of the unbound lipids and protein-free detergents micelles as well as the non-volatile salts. The protein micelle mass was expected to be around $170 \mathrm{kDa}$ (102.5 kDa for MgtA + ca. 65-70 kDa for the DDM micelle). MgtA was washed with freshly prepared aqueous ammonium acetate (AmAc) at a concentration of $200 \mathrm{mM}$ (adjusted with 
203

204

205

206

207

208

209

210

211

212

213

214

215

216

217

218

219

220

221

222

223

224

225

226

227

228

229

230

231

232

ammonium hydroxide to $\mathrm{pH} 7.0$ ), including 3x CMC DDM or $\mathrm{C}_{12} \mathrm{E}_{8} .25 \mu \mathrm{L}$ MgtA was added to $475 \mu \mathrm{L}$ washing solution in the cut-off concentrator column. The sample was concentrated back to $25 \mu \mathrm{L}$ (ca. $20 \mu \mathrm{M}$ protein, without $\mathrm{Mg}^{2+}$ by spinning it at $10,000 \mathrm{~g}$ at $4{ }^{\circ} \mathrm{C}$. When concentrated, the flow-through was discarded and $475 \mu \mathrm{L}$ fresh wash solution was added. Wash steps were repeated four times, after which the sample was placed in an Eppendorf tube on ice until measurement.

Native MS measurements were performed a Synapt G2 HD Q-TOF mass spectrometer (Waters, Wilmslow, UK) with travelling wave (T-wave) ion mobility, using nano-electrospray ionization (ESI) with gold-coated borosilicate capillaries prepared in-house. Instrument parameters in TOF mode were chosen to preserve noncovalent interactions while ensuring optimum spectral resolution: capillary voltage $2.0 \mathrm{kV}$; source temperature $30{ }^{\circ} \mathrm{C}$; sample cone $200 \mathrm{~V}$; extraction cone $2.0 \mathrm{~V}$; trap collision energy $150 \mathrm{~V}$; transfer collision energy $150 \mathrm{~V}$; trap bias $45 \mathrm{~V}$. Gas pressures in the instrument were: source 6.4 mbar; trap and transfer cell 0.02 mbar; IM cell $3.7 \cdot 10^{-4}$ mbar. Settings were optimized to provide desolvated signals while maintaining important non-covalent interactions.

\section{Nano Differential Scanning Fluorometry (nanoDSF)}

E. coli cardiolipin, specific cardiolipin species or POPE dissolved in chloroform were dried under an argon stream. The lipid film was resuspended in buffer (25 mM HEPES, pH 7.0; $200 \mathrm{mM} \mathrm{KCl}$; $5 \%$ glycerol and $\left.21 \times \mathrm{CMC}(1.9 \mathrm{mM}) \mathrm{C}_{12} \mathrm{E}_{8}\right)$ to a final concentration of $1.3 \mathrm{mM}$. MgtA (final concentration: $14 \mu \mathrm{M}$ ) was mixed with lipid in a 1:100 molar ratio in a final detergent concentration of $3 \mathrm{x} \mathrm{CMC} \mathrm{C}_{12} \mathrm{E}_{8}$ and filled up with buffer to $40 \mu \mathrm{L}$ sample volume. Samples were incubated at $4{ }^{\circ} \mathrm{C}$ for $16 \mathrm{~h}$, and afterwards excess lipids were removed by centrifugation (20000 g, $15 \mathrm{~min}, 4^{\circ} \mathrm{C}$ ). nanoDSF was performed using Prometheus NT.48 (Nanotemper) in a temperature range of $15-95{ }^{\circ} \mathrm{C}$ with a temperature gradient of $1{ }^{\circ} \mathrm{C}$ per minute. A minimum of triplicates was used for all experiments.

Protein melting temperatures were determined as maxima of the first derivative of the $350 \mathrm{~nm} / 330$ nm ratio determined by GraphPad Prism8 (www.graphpad.com).

\section{In vivo localization}


233 A plasmid carrying clover-mgtA was created by gene synthesis (Eurofin Genomics). A start codon 234 free $m g t A$ was connected to the $3^{\prime}$, end of a stop codon free clover by a linker (GGATCCGCTGGCTCCGCTGCTGGTTCTGGCGAATTCggatatc). The sequence is a Gly and

236 Ser rich flexible linker previously designed for GFP fusions (32).

237 This construct was inserted behind the $\mathrm{P}_{\text {araBAD }}$ promoter of pBAD33.1 (pBAD33.1 was a gift from 238 Christian Raetz; Addgene plasmid \#36267; http://n2t.net/addgene:36267; RRID:Addgene_36267) 239 (33), so that the ATG of the NdeI restriction site in pBAD33.1 is replaced by the clover start codon to create pSoA280. pSoA280 was transformed in E. coli MG1655, MG1655 clsABC::FRT 241 (MG1655 $\Delta c l s ;$ PO10), and Vibrio cholerae c6706. All bacteria were grown at $37^{\circ} \mathrm{C}$ in LuriaBertani (LB) medium with aeration. The media were supplemented with $50 \mu \mathrm{g} / \mathrm{mL}$ or $5 \mu \mathrm{g} / \mathrm{mL}$ chloramphenicol for E. coli or $V$. cholerae, respectively, to maintain the plasmid. Expression of Clover-MgtA fusion proteins was induced by addition of $0.2 \% \mathrm{~L}$-arabinose (Sigma) in exponential growth phase for $2 \mathrm{~h}$. A Deltavision Elite (GE Healthcare) microscope equipped with Deltavision CMOS camera, a climate chamber set to $37^{\circ} \mathrm{C}$, and an UPlanFLN 100x PH NA1.30 objective (Olympus) was used to image bacteria. $2 \mu \mathrm{L}$ induced bacterial culture was spotted on a $1 \%$ agar patches immediately prior to microscopy and images in the phase contrast as well as the GFP channel were acquired. The software Oufti (2015 Oufti, Jacobs-Wagner Lab) was used to detect bacteria in the phase contrast channel and overlay their outlines with a mesh that subdivides each cell in segments (34). This mesh was used to quantify the fluorescent intensity in each subdivision in the GFP channel. Intensity values and cell lengths were extracted from the Oufti output files using Matlab (R2019a, Mathworks) and used to plot the intensity profiles of cells. For better 254 visualization, the microscopic image were adjusted using the levels tool in Photoshop CS6 (Adobe) by setting the same black- and white-point values for all fluorescent images.

\section{SDS-PAGE and immunoblot}

$20 \mathrm{ml} \mathrm{LB}$ supplemented with appropriate concentrations of arabinose and chloramphenicol were 259 inoculated 1:100 with overnight culture. Cultures were grown for $2 \mathrm{~h}$ at $37^{\circ} \mathrm{C}$ with aeration. The

$260 \mathrm{OD}_{600}$ of the culture was measured and similar cell amounts were harvested by centrifugation at $261 \sim 20000 \mathrm{x}$ g at $4{ }^{\circ} \mathrm{C}$. Pellets were re-suspended in SDS loading buffer (50 mM Tris- $\mathrm{HCl}(\mathrm{pH} 6.8)$; $2622 \%$ sodium dodecyl sulfate (SDS); $100 \mathrm{mM}$ dithiothreitol (DTT); $10 \%$ glycerol, $0.02 \% \mathrm{NaN}_{3}$ ) 
263 and heated for $10 \mathrm{~min}$ at $90{ }^{\circ} \mathrm{C}$. The samples were separated by SDS-polyacrylamide gel 264 electrophoresis (PAGE) on $10 \%$ gels and transferred to nitrocellulose membranes (BioRad). 265 Clover was detected using monoclonal antibodies (anti-GFP 1:5000, Santa Cruz Biotechnologies) 266 and monoclonal anti mouse secondary antibodies conjugated to horseradish peroxidase (1:5000, 267 Bio-Rad). After incubation with ECL substrate (BioRad) chemiluminescence was recorded on a Bio-Rad ChemiDoc MP Imaging System.

\section{Flow cytometry}

$2715 \mathrm{ml} \mathrm{LB}$, supplemented with appropriate concentrations of arabinose and chloramphenicol, were 272 inoculated 1:100 with overnight culture. Cultures were grown for $2 \mathrm{~h}$ at $37^{\circ} \mathrm{C}$ with aeration. The $273 \mathrm{OD}_{600}$ of the culture was measured and the same amount of cells was harvested by centrifugation 274 at $20000 \mathrm{x}$ g at $4{ }^{\circ} \mathrm{C}$. Pellets were resuspended in PBS and FSC, SSC and fluorescence (EX 488 275 nm; EM 533 +/-30 nm) was rescored on a BD Accuri C6 Plus Flow Cytometer (BD Biosciences). 276 PBS was used as control to distinguish bacterial cells from other particles in FSC and SSC.

\section{Results}

Native mass spectrometry reveals selective binding of two cardiolipin molecules

280

281

282

283

284

285

286

287

288

289

290

291

Native mass spectrometry (MS) has recently emerged as a powerful tool for the investigation of dynamic and heterogeneous membrane protein structures and lipid interactions $(35,36)$. This approach was used in this study to characterize the stoichiometry of MgtA-lipid interations, and to investigate the selectivity of specific cardiolipin species binding preferentially to the protein.

Lipid interaction experiments were performed on purified protein, but full delipidation was not always achieved with the protocol (described in Material and Methods) with some samples showing low levels of retained native lipids. Two types of experiments were carried out: (i) a full E. coli lipid extract (Avanti Polar Lipids) was added to the purified protein in the presence of $3 \mathrm{x}$ CMC DDM in order to examine lipid binding selectivity, and (ii) a titration with cardiolipin (CL) was carried out to investigate binding affinity, using POPE as a nonspecific binding control. The E. coli lipid extract, used for MgtA lipidation, contains only $9.8 \% \mathrm{CL}$, while the majority of the lipid content consists of PE (57.5\%), PG (15.1\%) and uncharacterized components (17.6\%) 
(Avanti Polar Lipids). Cardiolipin used for titration experiments was extracted from E. coli membranes (Avanti Polar Lipids), containing a large variety of CL species with different acyl chain composition.

Figure 1a shows two charge states (m/z peaks) in the native mass spectrum of delipidated MgtA (as purified) and without addition of lipids; the corresponding full mass spectrum is shown in Supplementary figure 1. From this, the protein mass is determined experimentally as 102,585 +/$8 \mathrm{Da}$, which matches the calculated sequence mass for MgtA, i.e. 102,665.0 Da with and 102,533.8 Da without the N-terminal methionine, reasonably well. Figure $1 \mathrm{~b}$ shows the same charge states, using the same experimental conditions, after incubation of purified MgtA with E. coli lipid extract and subsequent detergent wash. Additional peaks are apparent now which suggests that lipid binding is detected, with the apparent mass shift for the first binding event calculated from these charge states as ca. $1380+/-200 \mathrm{Da}$, and for the second as ca. 1440 +/- $125 \mathrm{Da}$. These masses broadly correspond to the molecular weight of one and two cardiolipin molecules, respectively. It should be noted that other lipids present in the extract, in particular PE (719.3 Da) and PG (761.07 Da), have masses which are roughly half the mass of CL. It is therefore possible, at the limited mass resolution obtained on this instrument, that the observed lipid adduct peaks might be instead due to two or four bound PE or PG molecules, respectively. However, in that case the mass spectra would also be expected to show the $1 \mathrm{x}$ and $3 \mathrm{x}$ bound states, which is not the case. The identification of the bound lipids as cardiolipin species is also further corroborated by the following data.

We additionally performed a competition experiment in which binding of cardiolipin was studied in the presence of another lipid, POPE, added in large excess (Figure 2 and Supplementary figure 2). POPE is a major lipid component present in the E. coli membrane and, therefore, was used as a negative control representing the native E. coli lipid environment. Additionally, POPE was included to evaluate if hydrophobic exposed patches on the protein show any nonspecific lipid interactions in our setting. Lipid binding to MgtA was studied in the absence and presence of CL with increasing concentrations up to $30 \mu \mathrm{M}$, and correspondingly decreasing concentrations of POPE from 1000 to $970 \mu \mathrm{M}$, respectively. This leaves the total lipid concentration constant at $1000 \mu \mathrm{M}$, which corresponds to a 1:100 molar ratio between MgtA and lipid. In this experiment samples were obtained from a different protein batch, and even the control sample (first two panels in Figure 2a, Purified MgtA after lipid removal, and $1000 \mu \mathrm{M}$ POPE) revealed already a low 
amount of bound cardiolipin, which was most likely retained during the purification process in this case. These co-purified cardiolipins were not observed in the delipidated sample of the previous experiment (Figure 1), presumably due to differences in delipidation efficiency. Nevertheless, the addition of increasing amounts of CL showed a corresponding increase in the relative intensity of adduct peaks which correlate to those seen in Figure 1, with the relative intensity of the 1x bound state in particular increasing slightly from ca. $25 \%$ to ca. $30 \%$, for both the $25+$ and $24+$ charge states. Interestingly the $2 \mathrm{x}$ lipid-bound peak intensity seems to not change significantly within the limited concentration range studied here (Figure $2 \mathrm{~b}$ and Supplementary figure 2). While the data demonstrate qualitatively that the binding of the first CL lipid occurs with higher affinity and increases with increasing CL concentration, the second lipid binding event is of lower affinity and can only be seen clearly above $20 \mu \mathrm{M}$ CL (i.e. at a 2:1 ratio to the protein).

These results support that MgtA selectively binds up to two cardiolipin molecules, and not any other lipids present in the native $E$. coli membrane. They also show that the first binding event happens with higher affinity, and the mass of this cardiolipin species appears to be somewhat lower than that of the second one binding, although the achievable mass resolution and peak shapes do ultimately not allow to make firm conclusions. With a more powerful spectrometer, the masses of the individual lipids bound could be determined more accurately, and the lipid titration series presented here could also be extended to higher CL : POPE ratios in order to quantitatively determine binding affinities and possible cooperative effects.

\section{No specificity of cardiolipin binding to selected conformational states of MgtA was detected}

As a P-type ATPase, MgtA transports $\mathrm{Mg}^{2+}$ according to the catalytic cycle described by the PostAlbers-scheme (35). During the catalytic cycle MgtA likely alternates between different conformational states as described in the introduction for the $\mathrm{Na}^{+} / \mathrm{K}^{+}$-ATPase. The two major conformational states are termed E1 and E2, which exhibit large structural differences and different affinities to the transported ion and counter ion (35) (Supplementary figure 3A). To investigate whether MgtA has a higher affinity for cardiolipin in one specific conformational state, which would indicate a role of cardiolipin for a specific transport step, the protein was incubated with inhibitors $\mathrm{AlF}_{4}{ }^{-}$and $\mathrm{ADP}-\mathrm{AlF}_{4}{ }^{-}$. These inhibitors essentially mimic phosphate and $\mathrm{ATP}$ at the 
binding sites on the enzyme and lock MgtA in the two different conformational states, E1 and E2, respectively. After samples were incubated with inhibitors, CL extracted from the E. coli membrane (E. coli $\mathrm{CL}$ ) was added to MgtA as previously described. Native MS spectra showed binding of cardiolipin in all samples (Supplementary figure 3B). This indicates that there is no, or only a very small, preference of cardiolipin to a selected conformational state of MgtA. In summary, we find that MgtA contains two specific binding sites for lipids, and selectively binds cardiolipin among other major lipids present in E. coli membranes.

\section{The composition of the hydrophobic cardiolipin tail plays a major role in MgtA activation}

Cardiolipins are anionic lipids with a unique dimeric structure, with two phosphate ester groups and four acyl chains (Figure 3A). While the head group defines the lipid class, large molecular diversity is present through variations in the length and saturation degree of the fatty acid chains (Figure 3A). Previously, we have shown that cardiolipin is required for MgtA activation (11). However, all enzymatic assays were performed in the presence of cardiolipin extracted from $E$. coli membranes consisting of a mixture of different cardiolipin species (11). To investigate whether specific cardiolipins stimulate MgtA activity, cardiolipin species with varying acyl chain length and saturation were used in the ATPase assay. In E. coli, a large variety of cardiolipin species have been detected with over 50 different types present ranging in acyl carbon chain length from 12 to 19 containing different acyl chain combinations (30). Commercially only cardiolipins with four identical acyl chains are available at Avanti Polar Lipids, but these do not mimic the most common natural cardiolipin species in the E. coli membrane which contain mixed acyl chains. However, we used the CL species consisting of palmitic acid (16:0), palmitoleic acid (16:1) and oleic acid (18:1) as the most common acyl chains present in E. coli cardiolipin. Large differences in MgtA ATPase activity levels were detected in the presence of these different types of cardiolipin molecules (Figure 3B). For instance, activity was reduced to approximately $50 \%$ in the presence of CL 18:1 and to barely $10 \%$ in the presence of CL 14:1 in comparison to E. coli CL. In the presence of any single cardiolipin species, MgtA activity never reached the same activation level as in the presence of $E$. coli extracted cardiolipin.

Interestingly, an equimolar combination of two species, CL 18:1 and CL 16:0, exhibited higher MgtA ATPase activity levels relative to $E$. coli $\mathrm{CL}$ at the same protein:lipid ratio. It is noteworthy 
that although MgtA exhibits lower activity in the presence of CL 18:1 alone, CL 16:0 by itself does not seem to activate MgtA at all. This corresponds to previous results comparing MgtA activation in the presence of cardiolipin extracted from either E. coli or bovine heart membranes, which showed a decreased activity in the latter (14). While cardiolipins share the same head group, E. coli cardiolipin has a diverse fatty acid composition with 16:0-18:1 fatty acids at positions 1 and 2 of sn-glycerol 3-phosphate as the most dominant species (35\%), whereas bovine heart CLs contain almost entirely linoleate chains $(18: 1,18: 2,18: 3)$ and no stearic chains (16:0) (14).

As activity studies shown in figure 3B revealed the importance of two different cardiolipin species, CL 18:1 and CL 16:0 for MgtA activity, we hypothesized the specific interactions of these two lipid species with individual binding sites in MgtA. ATPase activity in the presence of selected cardiolipin species followed a sigmoidal shaped curve, which indicates cooperative binding at more than one binding site (Figure 3B). Cooperativity in protein-ligand interaction refers to increased affinity for additional ligands upon binding of the first ligand in the case of positive cooperativity, or decreased ligand affinity of the protein for additional ligands for negative cooperativity (37). Cooperativity of ligand binding can be quantified by the Hill coefficient $\left(\mathrm{n}_{\mathrm{H}}\right)$, which measures the sigmoidal character of the activation curve (38). The reference state is a hyperbolic curve with a single monomeric binding site, characterized by $\mathrm{n}_{\mathrm{H}}=1$. Therefore, binding curves with $\mathrm{n}_{\mathrm{H}}>1$ are a direct measurement for cooperativity. Although, $\mathrm{n}_{\mathrm{H}}$ is not a direct measurement of ligand binding sites, it is a useful index for the theoretical upper limit of the number of binding sites (38). Only recently, the first case of allosteric modulation and positive cooperative lipid binding has been detected for lipid-lipid interaction on E. coli ammonium channel AmtB (39). Fitting of the cardiolipin-dependent activation curve of MgtA to a sigmoidal allosteric model revealed positive cooperative binding of cardiolipin (Figure 3B; Table 1). In our analysis only least squares fits (R-square) of 0.95 or above were considered; i.e. only activation curves in the presence of $E$. coli CL and the CL 18:1 / CL 16:0 mixture in equimolar ratio. MgtA activation curves in the presence of CL 14:1, CL 18:1, CL 16:1 and CL 16:0 were excluded based on the poorer fit. Plotting of MgtA activation curves in the presence of E. coli CL, or equimolar amounts of CL 18:1 and CL 16:0 against an allosteric sigmoidal fit, showed a Hill coefficient of 2.4 - 3. This supports the hypothesis of more than one cardiolipin binding site with cooperative binding of multiple CL species. 


\section{Cardiolipin 16:0 is required for optimal MgtA activation}

414 To better understand the effect of CL 18:1 and 16:0 and their combination on MgtA activation, 415 ATPase activity profiles in the presence of these CL species with increasing $\mathrm{Mg}^{2+}$ concentrations 416 were performed. The total lipid concentration was kept constant at $12 \mathrm{mM}$ in all experiments. 417 Results confirmed previous observations that equimolar amounts of both lipid species lead to maximum activation (Figure 4A), mimicking the activity levels achieved previously in the

419 presence of native E. coli CL(14). In the presence of CL 18:1 alone, MgtA exhibited lower levels 420 of activity, while no measurable ATPase activity was observed in the presence of CL 16:0 alone 421 (Figure 4A). To assess whether the molar ratio between CL 18:1 and CL 16:0 affects activation 422 levels, ATPase assays were performed with molar ratios of 3:1 and 1:3, with either CL 18:1 or CL $42316: 0$ as the major lipid. Additionally a molar ratio of 1:1 between CL 18:1 and CL 16:0 (as above) 424 was included again. Maximum enzyme activity was obtained only when $50 \%$ or more of the lipid 425 content consisted of CL 16:0. Enzymatic assays with higher percentages of CL 18:1, e.g. in the $4263: 1$ ratio, resembled the activity levels obtained in the presence of CL 18:1 alone (Figure 4B).

427 To verify whether only either CL 18:1 or CL 16:0, mixed with another cardiolipin species, were 428 needed for optimal activation, ATPase assays in the presence of CL 18:1 or CL 16:0 mixed in a 429 1:1 molar ratio with CL 16:1 were performed (Figure 4C). Activation levels did not reach their maximum for samples containing CL 16:1 alone or in either combination with CL 16:0 or 18:1.

431 This highlights the importance of both species, CL 18:1 and CL 16:0, for MgtA activation. It should be noted though that only activating effects according to cardiolipin species were investigated, whereas possible variations in binding affinities between cardiolipin species were not assessed. As all experiments were however performed in large excess of CL at the same total concentration of $12 \mathrm{mM}$, we assume that if CL 16:0 would exhibit a much higher binding affinity than CL 18:1, it would occupy both binding sites, even in a sample with the majority of lipid represented by CL 18:1, and therefore MgtA would exhibit similar activity levels as in samples containing CL 18:1 and CL 16:0 in a 3:1 and 1:1 molar ratio.

439 Calculated $\mathrm{V}_{\max }, \mathrm{K}_{\mathrm{m}}$ and $\mathrm{k}_{\mathrm{cat}}$ in the presence of different cardiolipin species are given in Table 2. 440 Interestingly, higher $\mathrm{V}_{\max }$ and $\mathrm{k}_{\text {cat }}$ values were observed in the presence of CL 18:1 and CL 16:0 in equimolar amounts $\left(\mathrm{V}_{\max }: 19.3 \mu \mathrm{mol} \mathrm{min} \mathrm{mg}^{-1}, \mathrm{k}_{\mathrm{cat}}: 32.8 \mathrm{~s}^{-1}\right)$ or in excess of CL 16:0 ( $\mathrm{V}_{\max }$ : 
$24.6 \mu \mathrm{mol} \mathrm{min}{ }^{-1} \mathrm{mg}^{-1}, \mathrm{k}_{\mathrm{cat}}: 41.8 \mathrm{~s}^{-1}$ ) in comparison to previously obtained kinetic parameters in the presence of $E$. coli $\mathrm{CL}\left(\mathrm{V}_{\max }: 13.7 \mu \mathrm{mol} \mathrm{min} \mathrm{mg}^{-1}, \mathrm{k}_{\mathrm{cat}}: 23 \mathrm{~s}^{-1}\right)(14)$. However, $\mathrm{K}_{\mathrm{m}}$ values also increased for both samples (CL 18:1/16:0 1:1, $67 \mu \mathrm{M}$; CL 18:1/16:0 3:1, 120.9 $\mu \mathrm{M}$ ) in comparison to E. coli $\mathrm{CL}(10 \mu \mathrm{M})$, indicating decreased affinity for $\mathrm{Mg}^{2+}$ free. It should be noted that $\mathrm{K}_{\mathrm{m}}$ increased for all samples containing CL 16:0 or CL 16:1. Overall, results indicate an intricate interplay of lipid species CL 18:1 and CL 16:0 for maximum activity of MgtA.

\section{MgtA reveals increased thermal stability in the presence of specific cardiolipin species}

To determine the effect of cardiolipin on MgtA thermal stability, nano differential scanning fluorimetry (nanoDSF) was performed in the presence of E. coli CL or POPE in a 1:100 proteinlipid molar ratio, matching native MS experiments (Figure 5A). Melting temperatures revealed an increase of ca. $7^{\circ} \mathrm{C}$ in the presence of $E$. coli $\mathrm{CL}\left(\mathrm{T}_{\mathrm{m}}: 49.7^{\circ} \mathrm{C}\right)$ relative to purified MgtA without lipids. The presence of POPE $\left(\mathrm{T}_{\mathrm{m}}: 42.2{ }^{\circ} \mathrm{C}\right)$ did not show any upwards temperature shift and resembled the control of MgtA without lipids $\left(\mathrm{T}_{\mathrm{m}}: 41.8{ }^{\circ} \mathrm{C}\right)$ (Table 3). This indicates that cardiolipin specifically stabilizes MgtA, while the presence of any other lipid environment, represented by POPE as a major E. coli lipid component, does not lead to significant protein stabilization. To analyze whether specific cardiolipin species affect MgtA stability differently, nanoDSF was performed with CL 18:1, CL 16:0 and mixtures of both in different ratios (Figure 5B and $\mathrm{C}$ ). In the presence of CL 18:1, the melting temperature of MgtA was significantly increased by ca. $10{ }^{\circ} \mathrm{C}$, while no thermal stabilization was detectable in the presence of CL 16:0 alone (Figure 5B). Melting curves in the presence of both, CL 18:1 and CL 16:0, revealed increased stabilization when the higher proportion of CL 18:1 was present in the mixture (Figure 5C). While a 1:3 mixture, with CL 16:0 as the major component, or 1:1 CL 18:1/16:0 showed melting temperatures of 42.5 and $43.2^{\circ} \mathrm{C}$, respectively, in a 3:1 mixture with CL 18:1 as the major component, a melting temperature of $47^{\circ} \mathrm{C}$ was determined. This reflects previous results from the enzymatic studies, which revealed activation in the presence of CL 18:1 and CL 16:0, while in the presence of CL16:0 alone no activity was detectable. Interestingly, thermal stabilization of MgtA is detectable in the presence of CL 18:1 alone, while this single cardiolipin species was not enough for maximal enzymatic activation. 
471 As a control, thermal stabilization of MgtA in the presence of only the inhibitors, $\mathrm{AlF}_{4}^{-}$and ADP$472 \mathrm{AlF}_{4}^{-}$, was investigated, which revealed no effect (Figure 5D). It should be mentioned that melting 473 curves in the presence of E. coli CL, CL 18:1 and CL 18:1/16:0 mix showed a flattened curve in 474 comparison to the control without lipids. MgtA was incubated with the indicated lipids in excess 475 overnight (1:100 molar ratio between MgtA and lipid) and excess lipid was removed prior to the 476 experiment by centrifugation. In the case of E. coli CL, CL 18:1 and CL 18:1/16:0, some of the 477 protein was removed during the centrifugation step, likely pulled down together with the excess 478 lipid. This affected the final protein concentration in the experiment. The different curve shapes might therefore be due to lower protein concentrations or due to the presence of lipid vesicles 480 forming around the protein, which could interfere with the absorbance measured at 350 and 330 $\mathrm{nm}$ upon unfolding. Interestingly, the removal of protein by centrifugation of excess lipids already indicates binding of MgtA to the E. coli CL and CL 18:1. In summary, MgtA is selectively stabilized by a specific cardiolipin species, CL 18:1.

\section{Cardiolipin is not sole signal for localization of MgtA to cell poles}

486

487

488

489

490

491

492

493

494

495

496

497

498

499

500
Previously, we showed co-localization of a fluorescently-tagged MgtA to E. coli C43(DE3) cell poles (14). As C43(DE3) is an expression strain with genetic modifications in comparison to wild type E. coli (40), we verified here that fluorescently-tagged MgtA under the arabinose promoter (Figure 6A) also localizes to the cell poles in wild-type strain E. coli MG1655 (Figure 6B). Additionally, we could detect localization of MgtA to cell poles in cardiolipin-deficient strain MG1655 $\Delta c l s$ that contains a deletion of all three cardiolipin synthases (Figure 6B). To test whether the cardiolipin functions as a localization signal and the presence of cardiolipin is sufficient for MgtA localization to the cell poles, its localization was analysed in Vibrio cholerae. V. cholerae is a Gram-negative bacterium with a similar membrane composition as E. coli (41). However, it does not encode an endogenous MgtA homologue. Fluorescently-tagged MgtA did not localize to $V$. cholerae cell poles but distributed equally along the entire cell membrane (Figure 6C,D). To verify that the fluorescent signal is constituted by Clover-MgtA, a western blot directed against GFP investigating the expression of Clover-MgtA in E. coli and V. cholerae was performed. This control revealed that the fluorescence mainly derives from full-length Clover-MgtA in both bacterial strains, and degradation products or free GFP do not contribute to the fluorescent signal 
501 (Supplementary figure 4). In summary, these results indicate that MgtA is localized to bacterial cell poles through an unknown mechanism, which is, at least not solely, dependent on CL.

\section{Discussion}

505

506

507

508

509

510

511

512

513

514

515

516

517

518

519

520

521

522

523

524

525

526

527

528

529

Recent research has shown the importance of lipids for membrane protein function, affecting protein activity, stability, localization and oligomerization (42). However, one must distinguish effects through site-specific lipid-protein interactions in comparison to effects mediated through chemical and physical properties of the bilayer, e.g. membrane fluidity, tension and thickness or interfacial curvature (42). Lipid-protein interactions have long focused on different lipid classes, varying in their hydrophobic head group. Only recently, the high selectivity of proteins binding only to specific lipids with defined hydrophobic fatty acid tails has been detected.

In our work, we investigated lipid-membrane protein interactions of bacterial magnesium transporter MgtA and its highly specific binding of cardiolipin. We identified high specificity of MgtA for two cardiolipin species, CL 18:1 and CL 16:0. They are essential for enzymatic activity and thermal stabilization of MgtA. Further, native MS revealed two specific cardiolipin binding sites. These results highlight for the first time positive cooperative binding of two different species of the same lipid type leading to an activating effect on protein activity. However, as solely CL 18:1 mediated MgtA thermal stabilization, differences in the interaction between MgtA and both CL species and, therefore, potentially different roles of both lipid species for MgtA function can be hypothesized. As native MS revealed two cardiolipin binding sites and CL 18:1 has been shown to affect thermal stability, one can hypothesize that both binding sites are preferentially occupied by CL 18:1 leading to MgtA stabilization (Figure 7). CL 16:0 plays an essential role during MgtA ATPase activity in combination with CL 18:1. As activity assays are performed in large excess of cardiolipin, CL 16:0 might not interact directly with MgtA, but play an unknown role in the protein-lipid bilayer environment. However, this is purely speculative and the interaction between MgtA and both CL species requires further investigation.

Interestingly, E. coli membranes contain a large variety of cardiolipins with the most abundant species containing acyl chains 18:1, 16:1 and 16:0 (30). We highlight the importance of the combination of specific species CL 18:1 and CL 16:0 for MgtA activity, as their combination with 
another cardiolipin type, CL 16:1, exhibited severely decreased MgtA activity levels. Although higher $\mathrm{V}_{\max }$ and $\mathrm{k}_{\mathrm{cat}}$ was determined in the presence of cardiolipin species CL 18:1 and CL 16:0 in equimolar amounts in comparison to $E$. coli $\mathrm{CL}, \mathrm{K}_{\mathrm{m}}$ values also increased. This indicates lower affinity of MgtA to $\mathrm{Mg}^{2+}$ free in the presence of the specific cardiolipin species. However, higher $\mathrm{K}_{\mathrm{m}}$ values were always detected in the presence of CL 16:0 or CL 16:1. The more CL species with acyl chain length of 16 were present in the lipid mixture, the higher $\mathrm{K}_{\mathrm{m}}$ values were determined. Meanwhile, activity studies performed in the presence of CL 18:1 alone showed a $\mathrm{K}_{\mathrm{m}}$ of $12 \mu \mathrm{M}$, similar to the $\mathrm{K}_{\mathrm{m}}$ determined in the presence of $E$. coli $\mathrm{CL}(10 \mu \mathrm{M})$. As an anionic lipid cardiolipin binds $\mathrm{Mg}^{2+}$ and one might speculate that different cardiolipin species vary in their binding properties to $\mathrm{Mg}^{2+}$. CL has a propensity to form non-bilayer structures, proposed to form dynamic protein-lipid membrane domains (43). The phase behavior of CL has been shown to be dependent on divalent cations, including $\mathrm{Mg}^{2+}$ (44), and further acyl chain length and composition can affect lipid phase behavior (45). Therefore, it can be speculated that tested CL species exhibit small differences in their phase behavior, leading to variations in their interaction with $\mathrm{Mg}^{2+}$.

Although we have shown the importance of CL 18:1 and CL 16:0 for MgtA activity, the interaction between MgtA and these lipids is not completely understood yet. The molecular shape of acyl chains affect lipid packing and, therefore, membrane bilayer properties (46). Monounsaturated lipids such as CL 18:1 exhibit a kinked shape and tend to form fluid bilayers at physiological temperatures. Therefore, they exhibit higher flexibility and fluidity in comparison to lipids with saturated acyl chains (46). P-type ATPases undergo large conformational changes during their catalytic cycle, requiring flexibility regarding their lipid environment (47). Other members of the P-type ATPase family, including bacterial $\mathrm{Cu}^{2+}$ transporter CopA or heavy metal transporter ZntA, have shown higher activity levels upon increased lipid disorder and fluidity in the presence of unsaturated lipids (48-50). Additionally, MgtA exhibited activation in the presence of CL extracted from bovine heart membranes which contain mainly CL 18:2 (29). In summary, a preference of MgtA for CL 18:1 as an abundant monounsaturated lipid of the E. coli membrane corresponds to lipid interactions detected for other P-type ATPases.

In comparison, the interplay of CL16:0 and MgtA is more elusive and needs further investigation. As a lipid with saturated acyl chains, CL 16:0 exhibits different properties in comparison to monounsaturated lipids such as CL 18:1. Lipids with saturated acyl chains pack with higher 
560

561

562

563

564

565

566

567

568

569

570

571

572

573

574

575

576

577

578

579

580

581

582

583

584

585

586

587

588

589

densities and tend to form non-fluid gel phases (46), so one might speculate that CL 16:0 allows close packing of MgtA in lipid-detergent vesicles. In vivo the lipid bilayer exerts lateral pressure that supports structural integrity. However, during the purification process, the detergent solubilization decreases lateral pressure and likely increases conformational freedom of the protein (51). Enzymatic assays were not performed in liposomes, but instead in detergent-lipid micelles. Therefore, tighter packing of CL 16:0 might be beneficial for in vitro MgtA activation, but its function in vivo needs further investigation. Unfortunately, no additional saturated cardiolipin species could be included to investigate the effect of saturated lipids in MgtA-mediated ion transport, as only a limited selection of cardiolipins were commercially available at the time.

Further, cooperative binding of cardiolipin to MgtA was revealed, and the determined Hill coefficient of 2.4-3 in the presence of equimolar concentrations of CL 18:1 and CL 16:0 (Table 1) corresponds to the presence of two cardiolipin bining sites revealed by native MS. Allosteric modulation of protein-protein interactions by lipids has been previously described (39), with the E. coli ammonium channel AmtB binding two different lipid types, PE and CL (52). Additionally, it was shown that individual lipid binding affected allosteric interaction between AmtB and a soluble regulatory protein, GlnK. The allosteric modulation was highly selective regarding lipid head group and acyl chain composition. In both cases, it was proposed that binding of certain lipids stabilizes a specific conformation of AmtB which exhibits a higher binding affinity to PE or GlnK, respectively. One can speculate a similar mechanism for positive allosteric modulation of MgtACL binding as described for AmtB, as CL has been shown to stabilize MgtA. CL interaction at a specific binding site potentially stabilizes MgtA in a conformational state which exhibits increased affinity for the second CL molecule.

Interestingly, other members of the P-type ATPase family have shown high specificity towards lipids with a defined acyl chain composition. Their high lipid specificity has been linked to their requirement of high flexibility in the lipid bilayer due to the large conformational changes during the catalytic cycle (47). SERCA adapts to membranes of different hydrophobic thickness by inducing local deformations and a mismatch between hydrophobic thickness of the bilayer and its membrane embedded part for optimal flexibility (53) is required, with 1-Palmitoyl-2-oleoyl-snglycero-3-phosphocholine (16:0-18:1-PC) as the optimal lipid environment for SERCA. Shorter PC species (di-14:1-PC) were shown to surround the protein like a straitjacket, which prevented 
rotational and translational movement of the transmembrane helices (53). MgtA also exhibited decreased activation in the presence of the shorter cardiolipin species CL 14:1, likely induced through decreased flexibility required for conformational changes. It should be noted that, although we propose CL 16:0 plays a more indirect role for MgtA function in the protein-lipid bilayer environment, it cannot be excluded that the two identified cardiolipin binding sites are occupied with one from each cardiolipin species, one CL 18:1 and one CL 16:0. Small mass differences were identified between the first and second bound cardiolipin in the native MS spectrum (Figure 1), with the second bound CL revealing a slightly higher mass (ca. $1440+/-125$ $\mathrm{Da})$ in comparison to the first one $(1380+/-200 \mathrm{Da})$. The calculated masses for CL 18:1 which has four identical oleic acid chains, and CL 16:0 with four palmitic acid tails, are 1501.0 Da and 1396.9 Da, respectively, making it tempting to suggest that the first binding site is occupied by CL 16:0, while CL 18:1 binds preferentially to the second site. One could speculate that each cardiolipin species could match the hydrophobic thickness of a specific conformational state of MgtA during the catalytic cycle, therefore exhibiting different binding affinities in different states. However native MS did not reveal changes in cardiolipin binding of MgtA locked in different conformations in the presence of inhibitors (Supplementary Figure 3). The resolution of the native MS experiments was too low to distinctively distinguish different cardiolipin species. Therefore, additional native MS studies with higher resolution could allow identification of specific cardiolipin species bound to the two sites on MgtA. Further, native MS of lipid titration of CL 18:1 in the presence CL of 16:0, and vice versa, would allow determination of binding affinities of each cardiolipin species and could shed further light on the cooperative interaction between the lipids and the enzyme.

Other P-type ATPases also revealed site-specific lipid binding sites. For $\mathrm{Na}^{+} / \mathrm{K}^{+}$-ATPase, two distinct lipid binding sites have been detected (10). Interestingly, distinct properties were assigned for both sites. Lipid binding site A was connected to stabilization of the pump while lipid binding site B did not affect stabilization, but stimulated activity. Both effects are independent. We have no indication of distinct functions associated with the identified two cardiolipin binding sites on MgtA. As removal of cardiolipin strongly affected MgtA stability making native MS spectrum acquisition difficult, a role of both lipid sites for MgtA stability is likely. It should be noted that native MS was performed in DDM, whereas other studies replaced DDM with UDM which has a higher critical micelle concentration, and therefore allows acquisition of the spectra at lower 
621 activation energy (10). This might also allow obtaining spectra of less stable MgtA mutants.

622

623

624

625

626

627

628

629

630

631

632

633

634

635

636

637

638

639

640

641

642

643

644

645

646

647

648

649

650

Although native MS measurements revealed binding of two cardiolipin molecules, no indication of the localization of the lipid binding site on MgtA could be obtained. In future work, structural characterization of lipid-protein interaction by in silico or experimental methods, such as X-ray crystallography or cryo-EM, should be conducted to provide answers to the interaction sites and role of the lipids for MgtA function.

We also investigated co-localization of MgtA with cardiolipin at bacterial cell poles. Lipids have been linked to membrane protein localization, e.g. polar localization of osmosensory transporter ProP correlates with proportion and localization of cardiolipin and is less pronounced in $c s^{-}$cells (28). Therefore, cardiolipin has been proposed to promote ProP localization (28). Previous studies also showed co-localization of MgtA to cell poles with cardiolipin, proposing a promoting role of cardiolipin for MgtA localization (14). Our work confirmed polar localization of MgtA in E. coli wild-type strain MG1655. MgtA also localized at the cells poles in a cardiolipin synthase knockout strain, E. coli MG1655 $\triangle$ cls ABC. In this mutant strain no CL was detectable independent of the bacterial growth phase (55). However, full depletion of CL by genetic approaches induced increased PG concentrations at the cell poles. As PG has previously revealed activation of MgtA, although to a lesser extent than CL, MgtA could exhibit promiscuously a relatively high affinity for PG and display a pattern of cellular localization that is not influenced by depletion of CL (56). Therefore, no conclusion on the importance of cardiolipin for MgtA localization in E. coli can be assumed. Interestingly, in $V$. cholerae, which contains a similar membrane composition as E. coli, but no MgtA homologue, MgtA did not exhibit polar localization, but localized over the entire cell membrane. This highlights that cardiolipin does not function as the sole signal and promoter of polar MgtA localization. Further research must be performed to identify the mechanism for MgtA localization. A previously predicted signal peptide at the N-terminus, enriched in positively charged residues and hypothesized to interact with cardiolipin, was shown not to be involved in MgtA localization at the cell poles (14), but recent results imply a role of the N-terminus as a lipid anchor for MgtA (57).

In summary, our results reveal a complex interplay between MgtA and selective cardiolipin species, affecting MgtA activity, stability and localization. Insights obtained here contribute to our understanding of lipid specificity and lipid interaction of membrane proteins in general. 


\section{Acknowledgment}

653 We would like to thank John Crook and the Weibel lab for the donation of the E. coli strains 654 MG1655 and MG16554cls.

\section{Declaration of Interests}

657 The authors declare no Competing Financial or Non-Financial Interests.

\section{References}

660

661

662

663

664

665

666

667

668

669

670

671

672

673

674

675

676

677

678

679

680

681

682

683

684

685

686

1. Engelman DM (2005) Membranes are more mosaic than fluid. Nature 438(7068):578580 .

2. van Meer G, Voelker DR, \& Feigenson GW (2008) Membrane lipids: where they are and how they behave. Nat Rev Mol Cell Biol 9(2):112-124.

3. Corradi V, et al. (2019) Emerging Diversity in Lipid-Protein Interactions. Chem Rev 119(9):5775-5848.

4. Reading E, Laganowsky A, Allison TM, \& Robinson CV (2014) Membrane Proteins Bind Lipids Selectively To Modulate Their Structure And Function. Protein Science 23:231-231.

5. Laganowsky A, et al. (2014) Membrane proteins bind lipids selectively to modulate their structure and function. Nature 510(7503):172-175.

6. Mangialavori IC, et al. (2012) Autoinhibition mechanism of the plasma membrane calcium pump isoforms 2 and 4 studied through lipid-protein interaction. Biochem $J$ 443(1):125-131.

7. Cornelius F, Habeck M, Kanai R, Toyoshima C, \& Karlish SJ (2015) General and specific lipid-protein interactions in Na,K-ATPase. Biochim Biophys Acta 1848(9):17291743.

8. Cornelius F (2001) Modulation of Na,K-ATPase and Na-ATPase activity by phospholipids and cholesterol. I. Steady-state kinetics. Biochemistry-Us 40(30):88428851.

9. Habeck M, et al. (2015) Stimulation, inhibition, or stabilization of Na,K-ATPase caused by specific lipid interactions at distinct sites. J Biol Chem 290(8):4829-4842.

10. Habeck M, Kapri-Pardes E, Sharon M, \& Karlish SJ (2017) Specific phospholipid binding to Na,K-ATPase at two distinct sites. Proc Natl Acad Sci US A 114(11):29042909.

11. Contreras FX, Ernst AM, Wieland F, \& Brugger B (2011) Specificity of intramembrane protein-lipid interactions. Cold Spring Harb Perspect Biol 3(6). 
687 12. Hossain KR \& Clarke RJ (2019) General and specific interactions of the phospholipid bilayer with P-type ATPases. Biophys Rev 11(3):353-364.

13. Pohland AC \& Schneider D (2019) Mg2+ homeostasis and transport in cyanobacteria - at the crossroads of bacterial and chloroplast Mg2+ import. Biol Chem 400(10):1289-1301.

14. Subramani S, Perdreau-Dahl H, \& Morth JP (2016) The magnesium transporter A is activated by cardiolipin and is highly sensitive to free magnesium in vitro. Elife 5.

15. Groisman EA, et al. (2013) Bacterial Mg2+ homeostasis, transport, and virulence. Annu Rev Genet 47:625-646.

16. Bourret TJ, Liu L, Shaw JA, Husain M, \& Vazquez-Torres A (2017) Magnesium homeostasis protects Salmonella against nitrooxidative stress. Sci Rep 7(1):15083.

17. Cunrath O \& Bumann D (2019) Host resistance factor SLC11A1 restricts Salmonella growth through magnesium deprivation. Science 366(6468):995-+.

18. Apell HJ (2004) How do P-type ATPases transport ions? Bioelectrochemistry 63(12):149-156.

19. Bublitz M, Poulsen H, Morth JP, \& Nissen P (2010) In and out of the cation pumps: PType ATPase structure revisited. Current Opinion in Structural Biology 20(4):431-439.

20. Morth JP, et al. (2011) A structural overview of the plasma membrane $\mathrm{Na}+, \mathrm{K}+-\mathrm{ATPase}$ and H+-ATPase ion pumps. Nat Rev Mol Cell Biol 12(1):60-70.

21. Ford DC, Joshua GWP, Wren BW, \& Oyston PCF (2014) The importance of the magnesium transporter MgtB for virulence of Yersinia pseudotuberculosis and Yersinia pestis. Microbiology (Reading) 160(Pt 12):2710-2717.

22. Park M, Nam D, Kweon DH, \& Shin D (2018) ATP reduction by MgtC and $\mathrm{Mg}(2+)$ homeostasis by MgtA and MgtB enables Salmonella to accumulate RpoS upon low cytoplasmic $\operatorname{Mg}(2+)$ stress. Mol Microbiol 110(2):283-295.

23. Lin Z, et al. (2017) Virulence and Stress Responses of Shigella flexneri Regulated by PhoP/PhoQ. Front Microbiol 8:2689.

24. Chadani Y, et al. (2017) Intrinsic Ribosome Destabilization Underlies Translation and Provides an Organism with a Strategy of Environmental Sensing. Mol Cell 68(3):528-+.

25. Park SY, Cromie MJ, Lee EJ, \& Groisman EA (2010) A bacterial mRNA leader that employs different mechanisms to sense disparate intracellular signals. Cell 142(5):737748.

26. Gall AR, et al. (2016) Mg2+ regulates transcription of mgtA in Salmonella Typhimurium via translation of proline codons during synthesis of the MgtL peptide. Proc Natl Acad Sci US A 113(52):15096-15101.

27. Moncrief MB \& Maguire ME (1999) Magnesium transport in prokaryotes. J Biol Inorg Chem 4(5):523-527.

28. Romantsov T, et al. (2007) Cardiolipin promotes polar localization of osmosensory transporter ProP in Escherichia coli. Mol Microbiol 64(6):1455-1465.

29. Schlame M (2008) Cardiolipin synthesis for the assembly of bacterial and mitochondrial membranes. J Lipid Res 49(8):1607-1620.

30. Garrett TA, O'Neill AC, \& Hopson ML (2012) Quantification of cardiolipin molecular species in Escherichia coli lipid extracts using liquid chromatography/electrospray ionization mass spectrometry. Rapid Commun Mass Spectrom 26(19):2267-2274.

31. Subramani S \& Morth JP (2016) Heterologous Expression and Purification of the Magnesium Transporter A (MgtA) in Escherichia coli. Bio-protocol 6(22):e2001. 
32. Waldo GS, Standish BM, Berendzen J, \& Terwilliger TC (1999) Rapid protein-folding assay using green fluorescent protein. Nat Biotechnol 17(7):691-695.

33. Chung HS \& Raetz CR (2010) Interchangeable domains in the Kdo transferases of Escherichia coli and Haemophilus influenzae. Biochemistry-Us 49(19):4126-4137.

34. Paintdakhi A, et al. (2016) Oufti: an integrated software package for high-accuracy, highthroughput quantitative microscopy analysis. Mol Microbiol 99(4):767-777.

35. Konijnenberg A, van Dyck JF, Kailing LL, \& Sobott F (2015) Extending native mass spectrometry approaches to integral membrane proteins. Biol Chem 396(9-10):991-1002.

36. van Dyck JF, Konijnenberg A, \& Sobott F (2017) Native Mass Spectrometry for the Characterization of Structure and Interactions of Membrane Proteins. Methods Mol Biol 1635:205-232.

37. Neet KE (1995) Cooperativity in enzyme function: equilibrium and kinetic aspects. Methods Enzymol 249:519-567.

38. Edelstein SJ \& Le Novere N (2013) Cooperativity of allosteric receptors. J Mol Biol 425(9):1424-1432.

39. Patrick JW, et al. (2018) Allostery revealed within lipid binding events to membrane proteins. Proc Natl Acad Sci U S A 115(12):2976-2981.

40. Miroux B \& Walker JE (1996) Over-production of proteins in Escherichia coli: mutant hosts that allow synthesis of some membrane proteins and globular proteins at high levels. J Mol Biol 260(3):289-298.

41. Giles DK, Hankins JV, Guan Z, \& Trent MS (2011) Remodelling of the Vibrio cholerae membrane by incorporation of exogenous fatty acids from host and aquatic environments. Mol Microbiol 79(3):716-728.

42. Corradi V, et al. (2018) Lipid-Protein Interactions Are Unique Fingerprints for Membrane Proteins. ACS Cent Sci 4(6):709-717.

43. Mileykovskaya E \& Dowhan W (2009) Cardiolipin membrane domains in prokaryotes and eukaryotes. Biochim Biophys Acta 1788(10):2084-2091.

44. Vail WJ \& Stollery JG (1979) Phase changes of cardiolipin vesicles mediated by divalent cations. Biochim Biophys Acta 551(1):74-84.

45. Sankaram MB, Powell GL, \& Marsh D (1989) Effect of acyl chain composition on saltinduced lamellar to inverted hexagonal phase transitions in cardiolipin. Biochim Biophys Acta 980(3):389-392.

46. Ernst R, Ejsing CS, \& Antonny B (2016) Homeoviscous Adaptation and the Regulation of Membrane Lipids. J Mol Biol 428(24 Pt A):4776-4791.

47. Thogersen L \& Nissen P (2012) Flexible P-type ATPases interacting with the membrane. Curr Opin Struct Biol 22(4):491-499.

48. Autzen HE, et al. (2018) Interactions of a Bacterial Cu(I)-ATPase with a Complex Lipid Environment. Biochemistry-Us 57(28):4063-4073.

49. Zimmer J \& Doyle DA (2006) Phospholipid requirement and pH optimum for the in vitro enzymatic activity of the E. coli P-type ATPase ZntA. Biochim Biophys Acta 1758(5):645-652.

50. Moore BM, Lentz BR, \& Meissner G (1978) Effects of sarcoplasmic reticulum Ca2+ATPase on phospholipid bilayer fluidity: boundary lipid. Biochemistry-Us 17(24):52485255.

51. Palsdottir H \& Hunte C (2004) Lipids in membrane protein structures. Biochim Biophys Acta 1666(1-2):2-18. 
795

796

797
52. Cong X, Liu Y, Liu W, Liang X, \& Laganowsky A (2017) Allosteric modulation of protein-protein interactions by individual lipid binding events. Nat Commun 8(1):2203

53. Sonntag Y, et al. (2011) Mutual adaptation of a membrane protein and its lipid bilayer during conformational changes. Nat Commun 2:304.

54. Cohen E, et al. (2005) Purification of $\mathrm{Na}^{+}, \mathrm{K}+-\mathrm{ATPase}$ expressed in Pichia pastoris reveals an essential role of phospholipid-protein interactions. J Biol Chem 280(17):16610-16618.

55. Ryabichko S, et al. (2020) Cardiolipin is required in vivo for the stability of bacterial translocon and optimal membrane protein translocation and insertion. Sci Rep 10(1):6296.

56. Oliver PM, et al. (2014) Localization of anionic phospholipids in Escherichia coli cells. $J$ Bacteriol 196(19):3386-3398.

57. Jephthah S, Mansson LK, Belic D, Morth JP, \& Skepo M (2020) Physicochemical Characterisation of KEIF-The Intrinsically Disordered N-Terminal Region of Magnesium Transporter A. Biomolecules 10(4).

\section{Figure 1}

a MgtA after stringend lipid removal

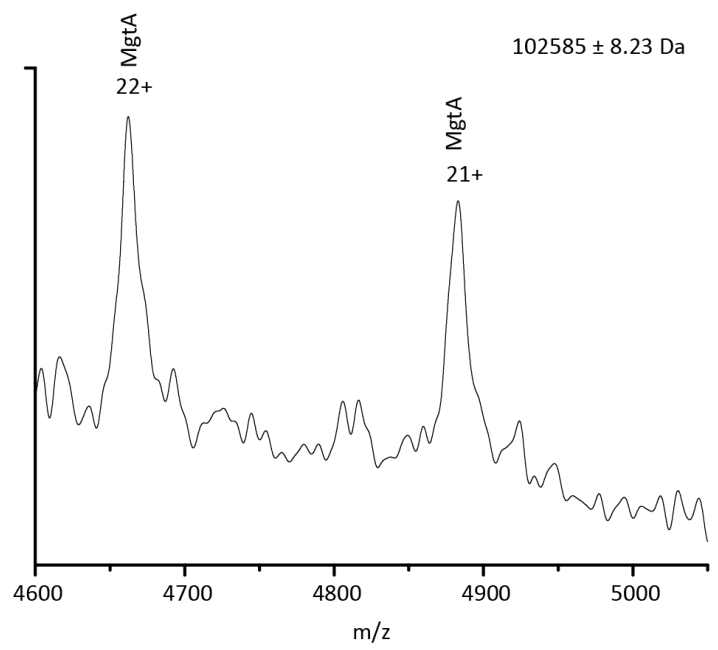

b Added Ecoli lipid mix after stringent lipid removal

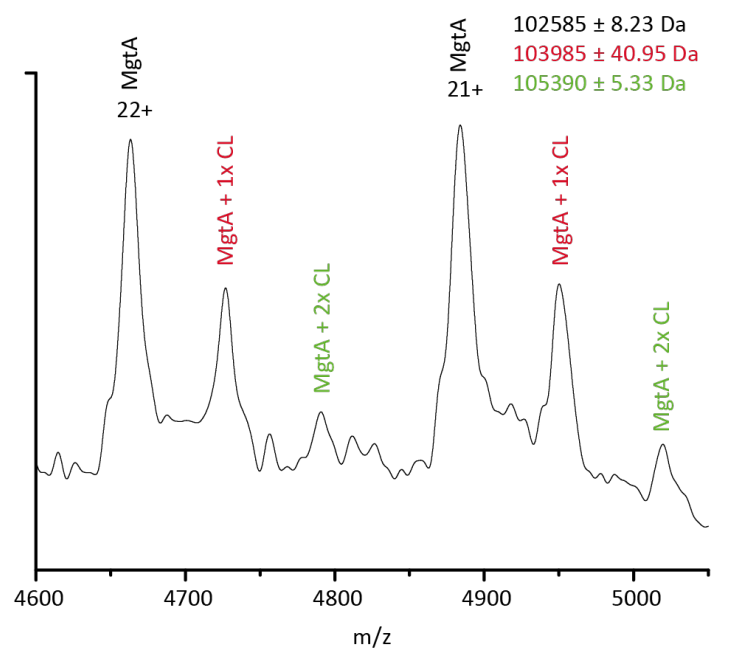

$\mathrm{m} / \mathrm{z}$ difference

\begin{tabular}{|l|l|l|}
\cline { 2 - 3 } \multicolumn{1}{c|}{} & $22+$ & $21+$ \\
\hline MgtA $>$ MgtA + 1x CL & $62.54 \pm 7.35$ & $66.17 \pm 10,83$ \\
MgtA + 1x CL >MgtA + 2x CL & $65.32 \pm 5.03$ & $68.88 \pm 6,97$ \\
\hline
\end{tabular}

\begin{tabular}{|l|l|l|}
\cline { 2 - 3 } \multicolumn{1}{c}{} & \multicolumn{2}{c}{ mass difference (Da) } \\
\cline { 2 - 3 } \multicolumn{1}{c|}{$22+$} & $21+$ \\
\hline MgtA $>$ MgtA $+1 \times C L$ & $1375 \pm 161$ & $1389 \pm 238$ \\
\hline
\end{tabular}




\section{Figure 1: MgtA shows selective binding of cardiolipin in native mass spectrometry}

800 Purified MgtA solubilized in 3x CMC DDM was analysed by native MS (a) in the absence or (b) presence 801 of total E. coli lipid extract. Total E. coli lipid extract contains only $9.8 \%$ CL, while the remaining lipids 802 are $57.5 \%$ PE, $15.1 \%$ PG and $17.6 \%$ unknown (Avanti Polar Lipids). The left spectrum shows the most 803 intense charge states $22+$ and $21+$ of purified MgtA after lipid removal by high CMC DDM. The right 804 spectrum exhibits MgtA as seen after incubation with $E$. coli lipid mixture. This sample was washed with $8053 x$ CMC DDM to remove weakly bound lipids leaving only the strongest interacting lipids. The spectrum 806 on the right reveals additions of approximately 1400 and $2800 \mathrm{Da}$ (red and green) which correspond to the 807 molecular weight of one and two cardiolipin molecules, respectively. The spectra were recorded in TOF 808 mode and the key MS settings are the voltages (sampling cone, trap collision, transfer collision and trap 809 bias) and pressures (backing pressure, trap cell pressure). These values were $200 \mathrm{~V}, 150 \mathrm{~V}, 150 \mathrm{~V}, 45 \mathrm{~V}$, 8106.41 mbar and $2.00 \times 10^{-2}$ mbar respectively. 
Figure 2

a Purified MgtA after lipid removal

$1000 \mu \mathrm{M}$ POPE

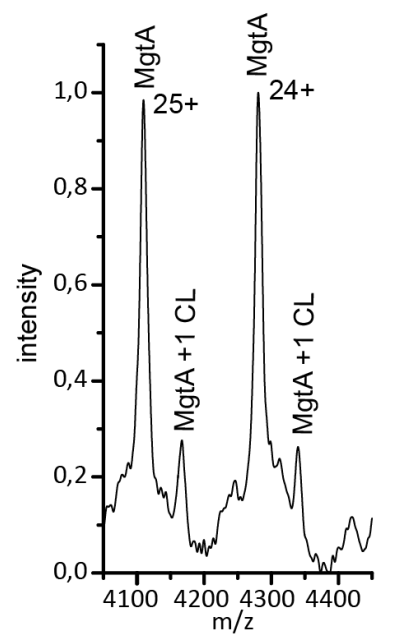

$990 \mu \mathrm{M}$ POPE $/ 10 \mu \mathrm{M} \mathrm{CL}$
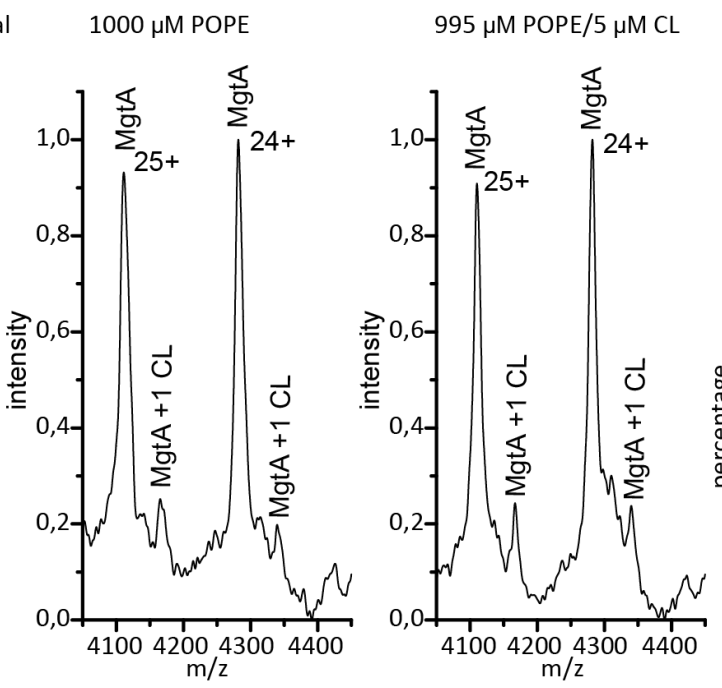

$980 \mu \mathrm{M}$ POPE $/ 20 \mu \mathrm{M} \mathrm{CL}$

$970 \mu \mathrm{M}$ POPE $/ 30 \mu \mathrm{M} \mathrm{CL}$
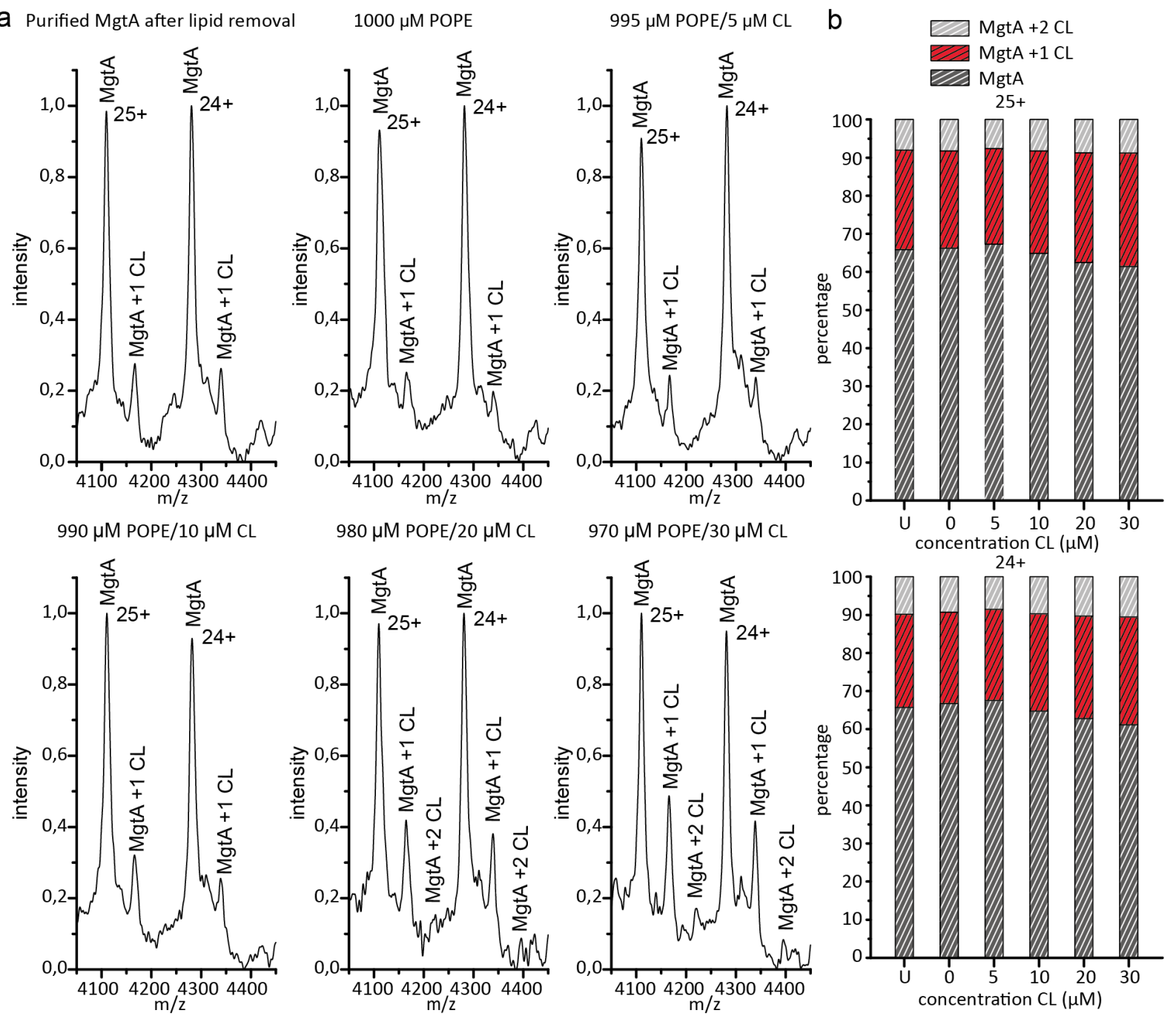

Figure 2: Cardiolipin competition assay of MgtA in the presence of POPE reveals increase in CL binding

a) MS spectra showing a lipid competition assay of MgtA with increasing CL concentrations (0 to $30 \mu \mathrm{M})$ and 1000 to $970 \mu \mathrm{M}$ POPE. In the spectra $1 \mathrm{x}$ and $2 \mathrm{x}$ CL adducts are assigned. In $\mathbf{b})$ the increase in $1 \mathrm{x}$ or 2x CL molecules bound to MgtA is shown as percentages according to the increase in CL added to the sample. The spectra were recorded in TOF mode and the key MS settings are the voltages: sampling cone, trap collision, transfer collision, trap bias and pressures: backing pressure, trap cell pressure. These values where $150 \mathrm{~V}, 200 \mathrm{~V}, 5 \mathrm{~V}, 45 \mathrm{~V}, 8.41 \mathrm{mbar}$ and $2.66 \times 10^{-2} \mathrm{mbar}$. 
Figure 3

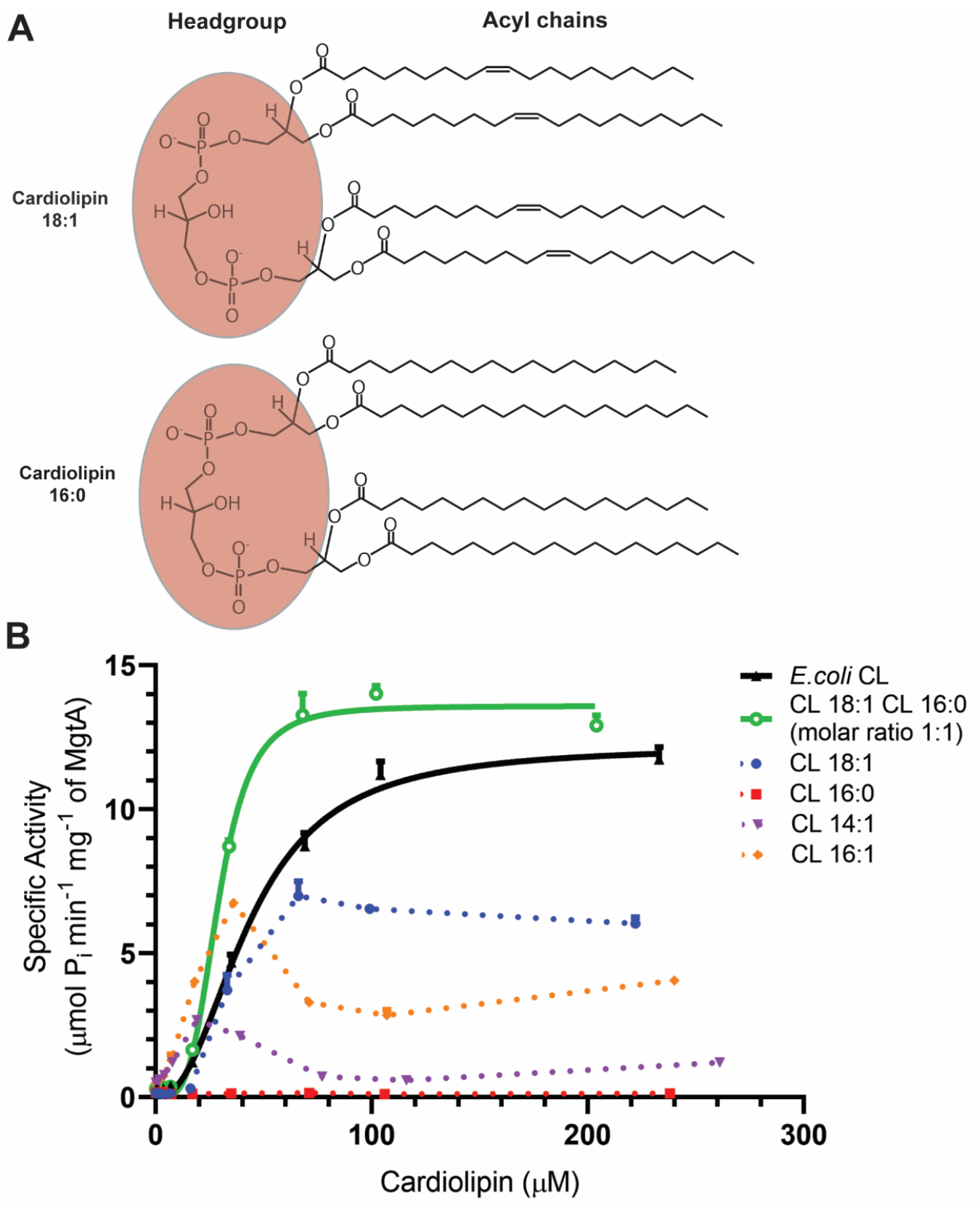

Figure 3: $E$. coli MgtA shows cooperative binding of specific cardiolipin species

(A) Cardiolipins 18:1 and 16:0 contain the same polar hydrophilic headgroup (red), but exhibit differences in the length and saturation degree of their acyl chains.

833 (B) Concentration-dependent activation of MgtA ATPase activity by E. coli extracted cardiolipin (black), a 1:1 mixture of cardiolipin 18:1 and 16:0 (green) or individual cardiolipin species. Lipids are prepared as described in the Materials and Methods section; specific activity is determined by measuring phosphate release. Curves are representatives from three independent experiments, showing mean +/- SD. Enzymatic activation curves were fitted to an allosteric sigmoidal model (GraphPad, Prism8). Fitting curves are shown 
Table 1: Allosteric fit of purified MgtA in the presence of different cardiolipin species. The $\mathrm{V}_{\max }$ and Hill coefficient were determined by least squares fit of the data from Figure 1B, as described in materials and methods. Only data from least squares fit of 0.95 or above was included in the analysis.

\begin{tabular}{|c|c|c|}
\hline & 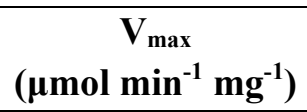 & Hill coefficient \\
\hline E. coli $\mathrm{CL}$ & $11.0+/-0.8$ & $2.4+/-0.6$ \\
\hline $\begin{array}{l}\text { CL 18:1 CL 16:0 } \\
\text { (molar ratio 1:1) }\end{array}$ & $14.1+/-1.9$ & $3.0+/-0.8$ \\
\hline
\end{tabular}



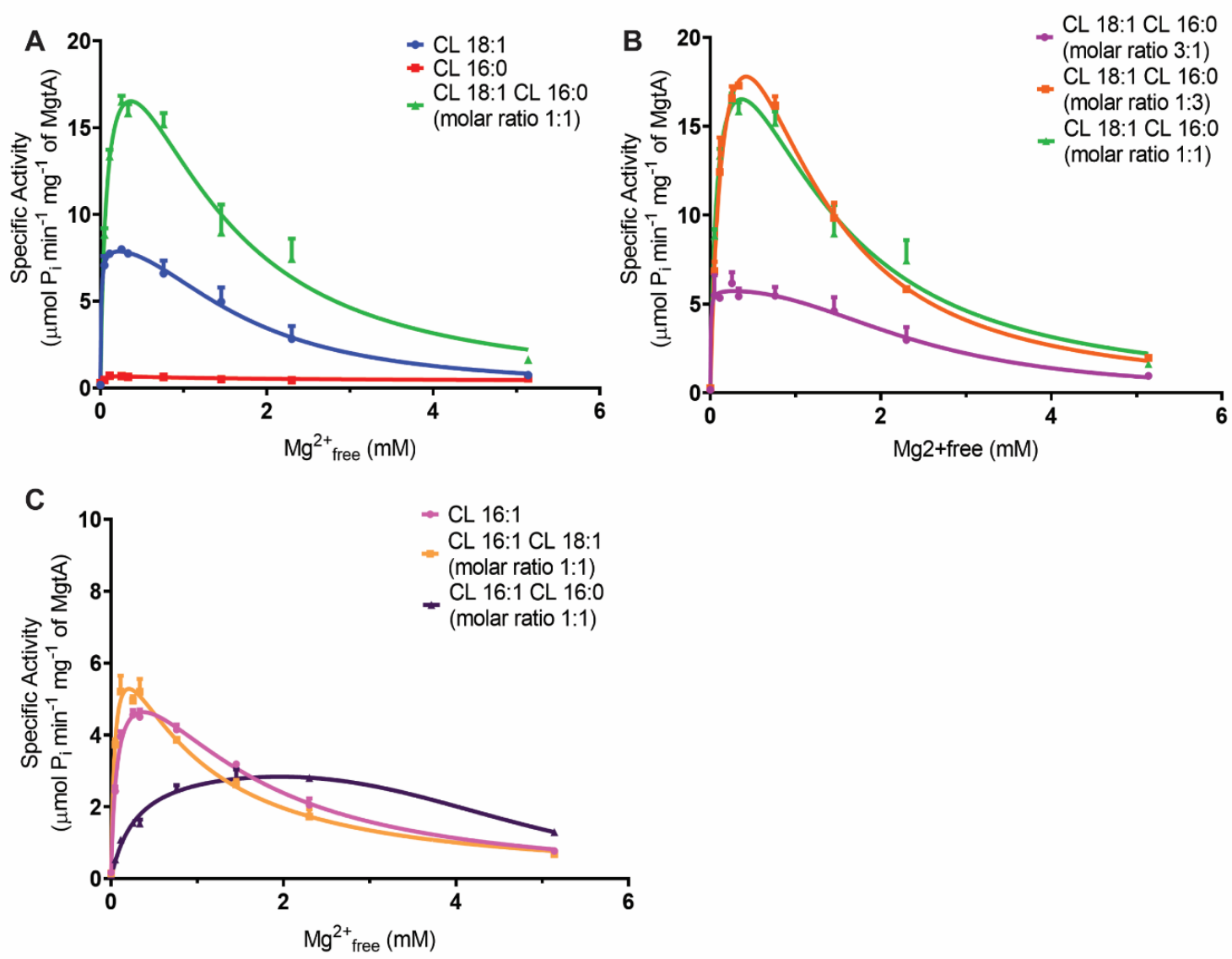

Figure 4: $E$. coli MgtA shows selective activation by cardiolipin species 18:1 and 16:0 in specific molar ratios

867 ATP hydrolysis was measured in the presence of specific cardiolipin species with increasing concentrations of $\mathrm{Mg}^{2+}$ at $3 \mathrm{mM}$ ATP. Lipid concentration was kept constant at $12 \mathrm{mM}$ (molar ratio of MgtA to lipid 1:5000). Curves are representatives from three independent experiments, showing mean +/- SD. Enzymatic data was fitted as a function of $\mathrm{Mg}^{2+}$ free using Michaelis-Menten equation (Graphpad Prism 8) as described in the method section. Kinetic parameters are described in table $2 . \mathrm{Mg}^{2+}$ free profiles of (A) CL 18:1, CL 16:0

872 and an equimolar mixture of both or (B) CL 18:1 and CL 16:0 in different molar ratios was assessed. (C) As a control $\mathrm{Mg}^{2+}$ free profiles of CL 18:1 or CL 16:0 in combination with CL 16:1 were performed. 
Table 2: Kinetic property of purified MgtA in the presence of different cardiolipin species. The apparent $\mathrm{V}_{\max }, \mathrm{K}_{\mathrm{m}}$ and the turnover number $\left(\mathrm{K}_{\mathrm{cat}}\right)$ values were determined by least squares fit of the data

878 from figure 2A, 2B and 2C and put in references to previously obtained kinetics (Subramani et al. (2016)).

\begin{tabular}{|c|c|c|c|}
\hline & $V_{\max }\left(\mu \mathrm{mol} \min ^{-1} \mathrm{mg}^{-1}\right)$ & $K_{m}(\mu M)$ & $K_{\text {cat }}\left(s^{-1}\right)$ \\
\hline $\begin{array}{c}\text { E. coli CL } \\
\text { (Subramani et al. (2016)) }\end{array}$ & $13.7+/-0.2$ & $10+/-0.6$ & $23+/-0.3$ \\
\hline CL 18:1 & $7.6+/-0.7$ & $12+/-7.0$ & $12.8+/-1.2$ \\
\hline CL 16:0 & $\mathrm{N} / \mathrm{A}$ & $\mathrm{N} / \mathrm{A}$ & $\mathrm{N} / \mathrm{A}$ \\
\hline $\begin{array}{c}\text { CL 18:1 CL 16:0 } \\
\text { (ratio 1:1) }\end{array}$ & $19.3+/-2.4$ & $67+/-3.0$ & $32.8+/-4.1$ \\
\hline $\begin{array}{c}\text { CL 18:1 CL 16:0 } \\
\text { (ratio 3:1) }\end{array}$ & $7.1+/-0.9$ & $4.4+/-2.6$ & $12.06+/-1.5$ \\
\hline $\begin{array}{c}\text { CL 18:1 CL 16:0 } \\
\text { (ratio 1:3) }\end{array}$ & $24.6+/-6.7$ & $120.9+/-48.9$ & $41.8+/-11.4$ \\
\hline CL 16:1 & $6.0+/-0.5$ & $103+/-5$ & $10.2+/-0.8$ \\
\hline $\begin{array}{c}\text { CL 18:1 CL 16:1 } \\
\text { (ratio 1:1) }\end{array}$ & $6.6+/-0.8$ & $40+/-12$ & $11.3+/-1.4$ \\
\hline $\begin{array}{l}\text { CL 16:0 CL 16:1 } \\
\text { (ratio 1:1) }\end{array}$ & $3.9+/-0.5$ & $285+/-44$ & $6.7+/-0.8$ \\
\hline
\end{tabular}

879

880

881 

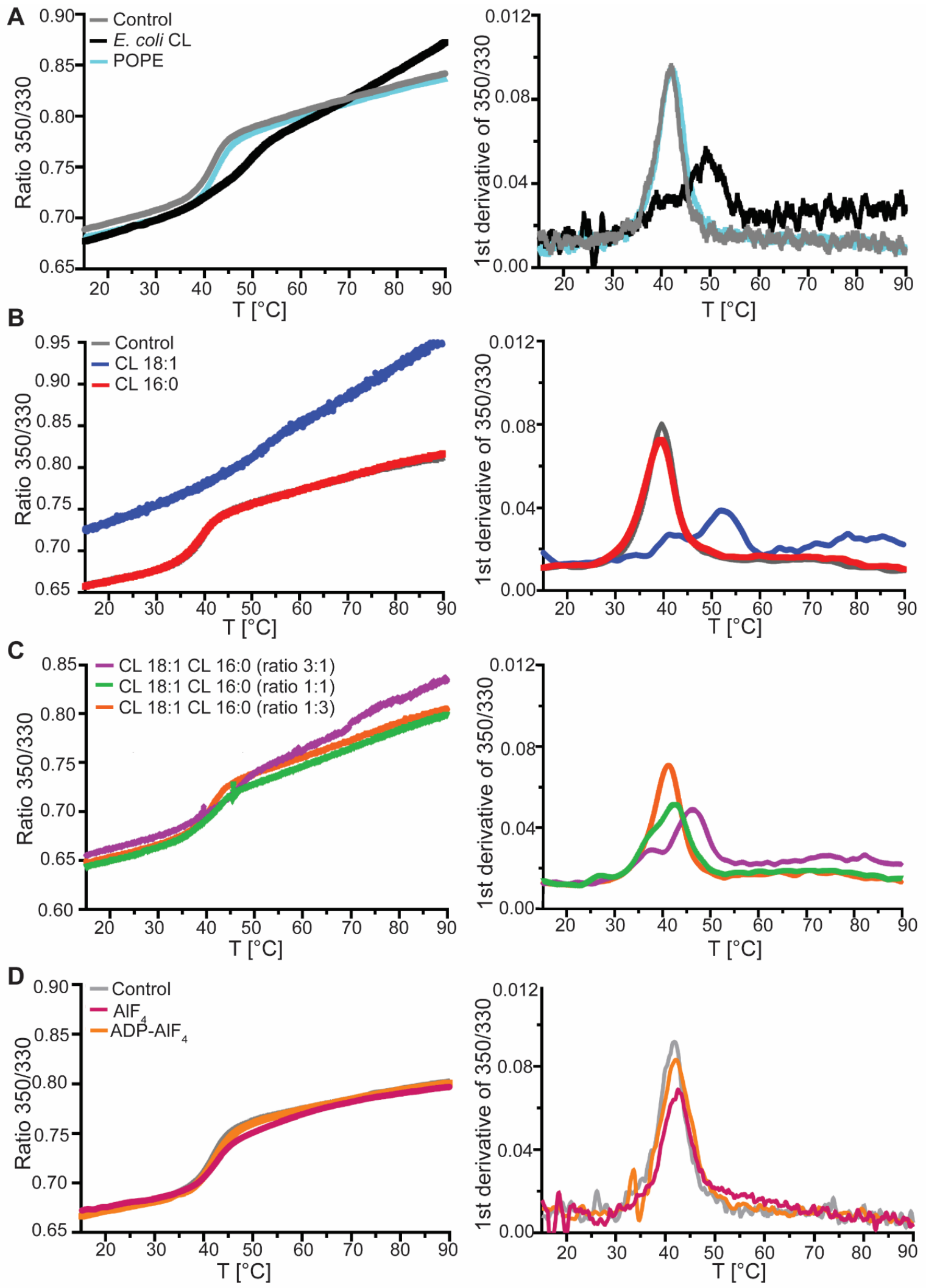
Figure 5: MgtA shows increased temperature stabilization in the presence of the specific cardiolipin species, cardiolipin 18:1

Nano differential scanning fluorimetry (nanoDSF) curves (left) and their first derivative (right) of purified MgtA in the presence of (A) cardiolipin and 1-Palmitoyl-2-oleoyl-sn-glycero-3-phosphoethanolamine (POPE) or specific cardiolipin species CL 18:1 and CL 16:0 (B) alone and (C) in different molar ratios were obtained. MgtA was incubated overnight with indicated lipids in a 1:100 molar ratio and samples were centrifuged to remove excess of lipids. Melting curves were performed on Prometheus NT.48 (Nanotemper) in a temperature range of $15^{\circ} \mathrm{C}-95^{\circ} \mathrm{C}$ and a temperature gradient of $1{ }^{\circ} \mathrm{C}$ per minute. A paired, one-sided t-test was applied. $* * * p<0.001$. (D) Additionally, melting curves were performed on MgtA in the presence of inhibitors, $\mathrm{ADP}_{-} \mathrm{AlF}_{4}{ }^{-}$and $\mathrm{AlF}_{4}^{-}$. MgtA was incubated with inhibitors in 1:500 molar ratio $(2.95 \mathrm{mM})$ for two hours at $4{ }^{\circ} \mathrm{C}$ before melting curves were performed. Curves are representatives from three independent

mean

$895+/-$ SD. Obtained melting temperatures are described in table 3.

Table 3: Melting temperatures of MgtA in the presence of lipids and inhibitors determined by

\begin{tabular}{|l|l|}
\hline & Melting temperature $\left.{ }^{\circ}{ }^{\circ} \mathbf{C}\right]$ \\
\hline MgtA (control) & $41.8+/-0.4$ \\
\hline MgtA in presence of E. coli cardiolipin & $49.7+/-1.4 * * *$ \\
\hline MgtA in presence of POPE & $42.2+/-0.4$ \\
\hline MgtA in presence of cardiolipin 18:1 & $52.5+/-1.2 * * *$ \\
\hline MgtA in presence of cardiolipin 16:0 & $40.3+/-1.0$ \\
\hline $\begin{array}{l}\text { MgtA in presence of cardiolipin 18:1 16:0 } \\
\text { (molar ratio 3:1) }\end{array}$ & $47.0+/-0.8$ \\
\hline $\begin{array}{l}\text { MgtA in presence of cardiolipin 18:1 16:0 } \\
\text { (molar ratio 1:1) }\end{array}$ & $43.2+/-1.2$ \\
\hline $\begin{array}{l}\text { MgtA in presence of cardiolipin 18:1 16:0 } \\
\text { (molar ratio 1:3) }\end{array}$ & $42.5+/-1.2$ \\
\hline MgtA in presence of AlF $_{4}^{-}$ & $41.8+/-0.6$ \\
\hline MgtA in presence of $\mathbf{A D P}_{-} \mathbf{A l F}_{4}^{-}$ & $41.6+/-0.6$ \\
\hline
\end{tabular}



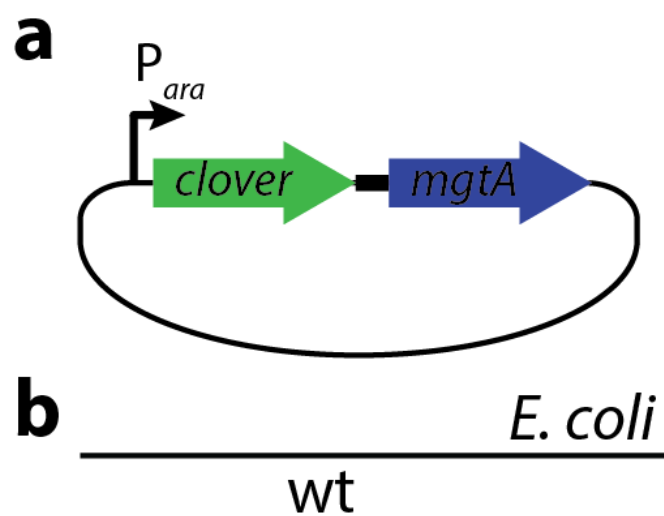

$\mathrm{PH}$ clover-mgtA
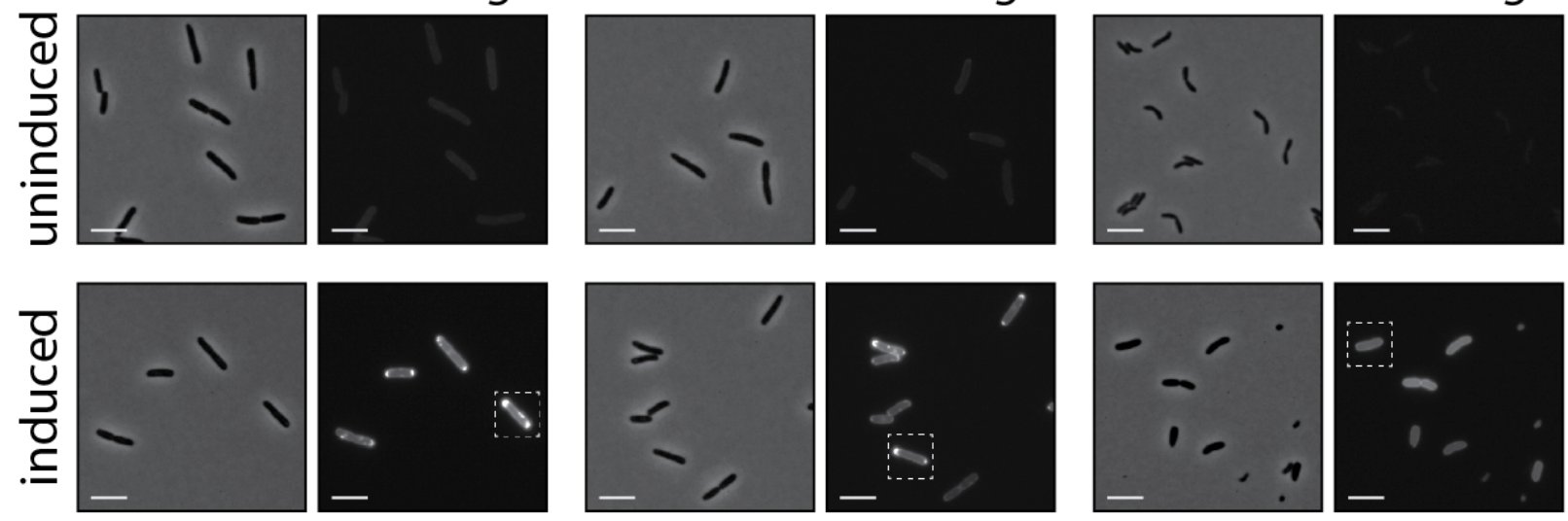

C
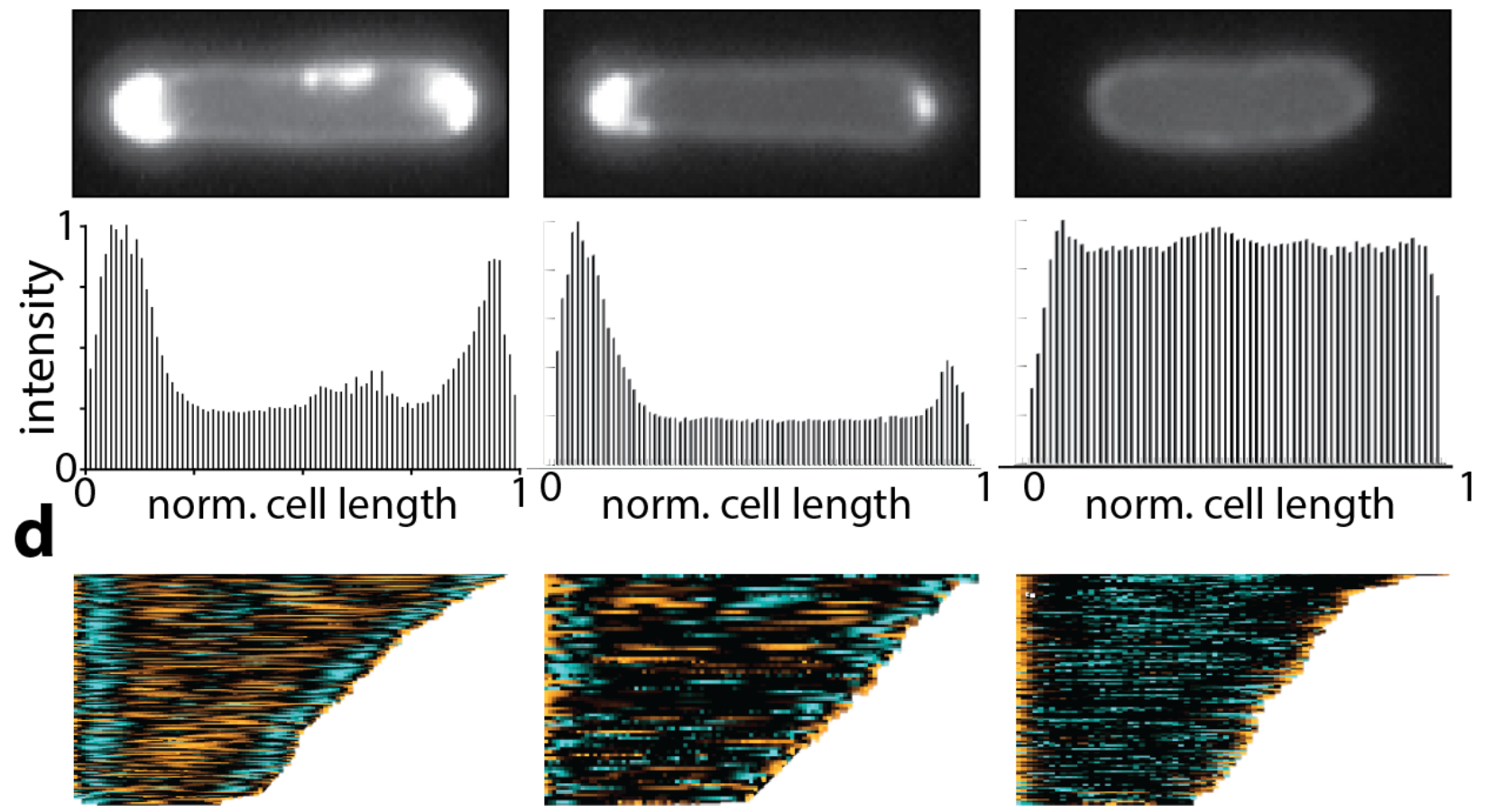
Figure 6: MgtA localization to the pole is not solely cardiolipin-dependent

905

906

907

908

909

910

911

912

913

914

915

916

917

918

919

920

921

922

To assess whether cardiolipin functions as the main signal for polar MgtA localization in E. coli, in vivo imaging studies were performed using a GFP-tagged MgtA construct.

(A) Schematic representation of the Clover-MgtA expression plasmid. The expression is controlled by the arabinose-inducible $\mathrm{P}_{\text {ara }}$ promoter.

(B) The localization of MgtA-GFP was assessed in wild-type E. coli strain MG1655, cardiolipin-deficient E. coli MG1655 $4 c l s$, a knockout strain of cardiolipin synthase $(\Delta c l s)$, and wild type Vibrio cholerae strain C6706. The phase contrast channel (PH) and GFP channel (Clover-MgtA) of bacteria grown in the presence of $0.2 \%$ arabinose (induced) or in its absence (uninduced) are shown. White scale bars are $5 \mu \mathrm{m}$.

(C) Intensity profiles of single, representative cells (highlighted by a dotted white box in (B)). The intensities are normalized to the brightest area in each cell, respectively.

(D) Clover-MgtA localization profiles of cells sorted by their cell length. This representation approximates the localization patterns through the cell cycle with small cells approximating newborn cells and long cells approximating cells immediately prior to division. The cell lengths are normalized to the longest cell in each analysis and the fluorescent intensities are normalized to the brightest area in all cells from each strain. E. coli MG1655 ( $\mathrm{n}=171$ cells), E. coli MG1655 $\Delta$ cls $(\mathrm{n}=75$ cells), and $V$. cholerae $\mathrm{C6706}$ ( $\mathrm{n}=125$ cells).

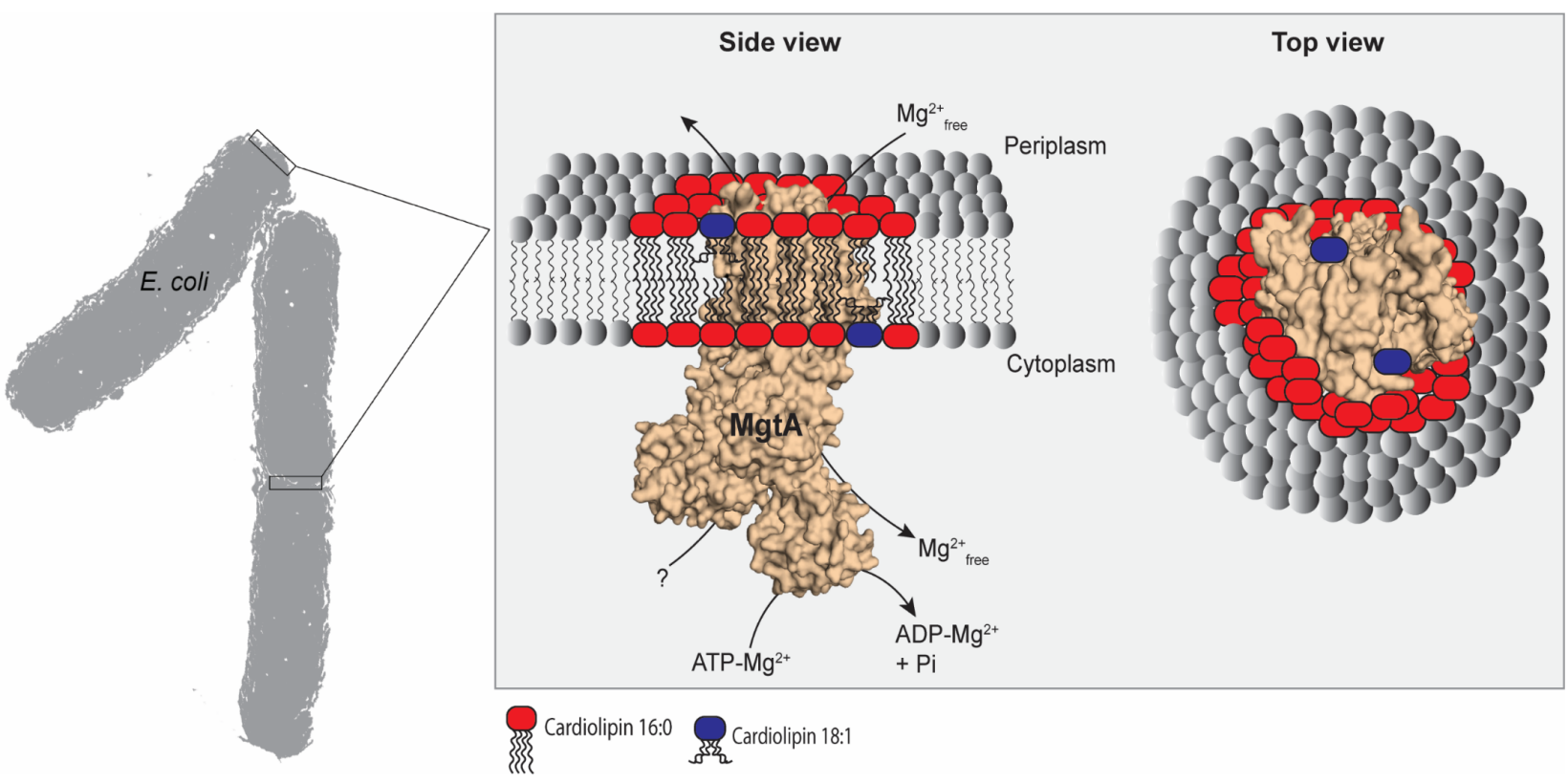

\section{Figure 7: Model of cardiolipin interaction with MgtA}

MgtA protein localizes in the inner membrane at the E. coli cell poles, which represent cardiolipin-rich regions. Association of MgtA with cardiolipin is essential for MgtA activity and stability, but MgtA shows high selectivity for specific cardiolipin species. We propose specific interaction between MgtA and two CL 18:1 molecules, affecting MgtA thermal stabilization. Further, CL18:1 is required for MgtA ATPase 


\section{Supplementary figures}

a
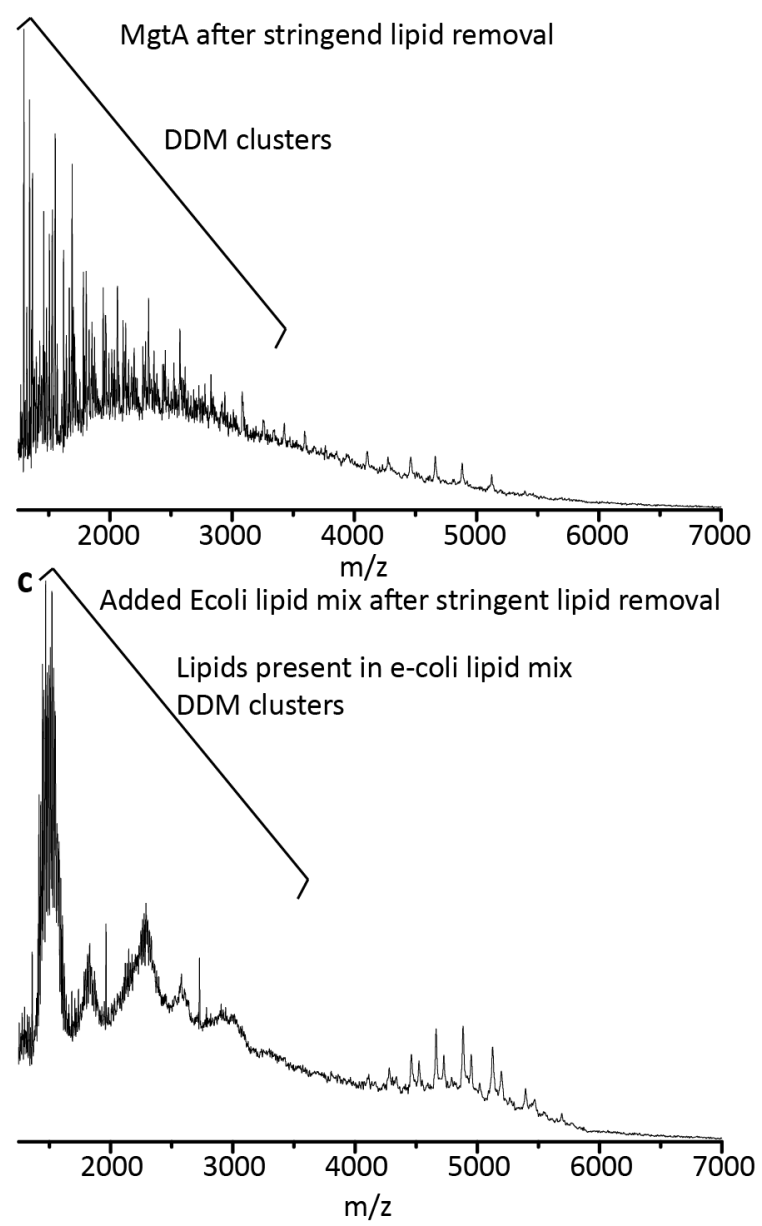

b
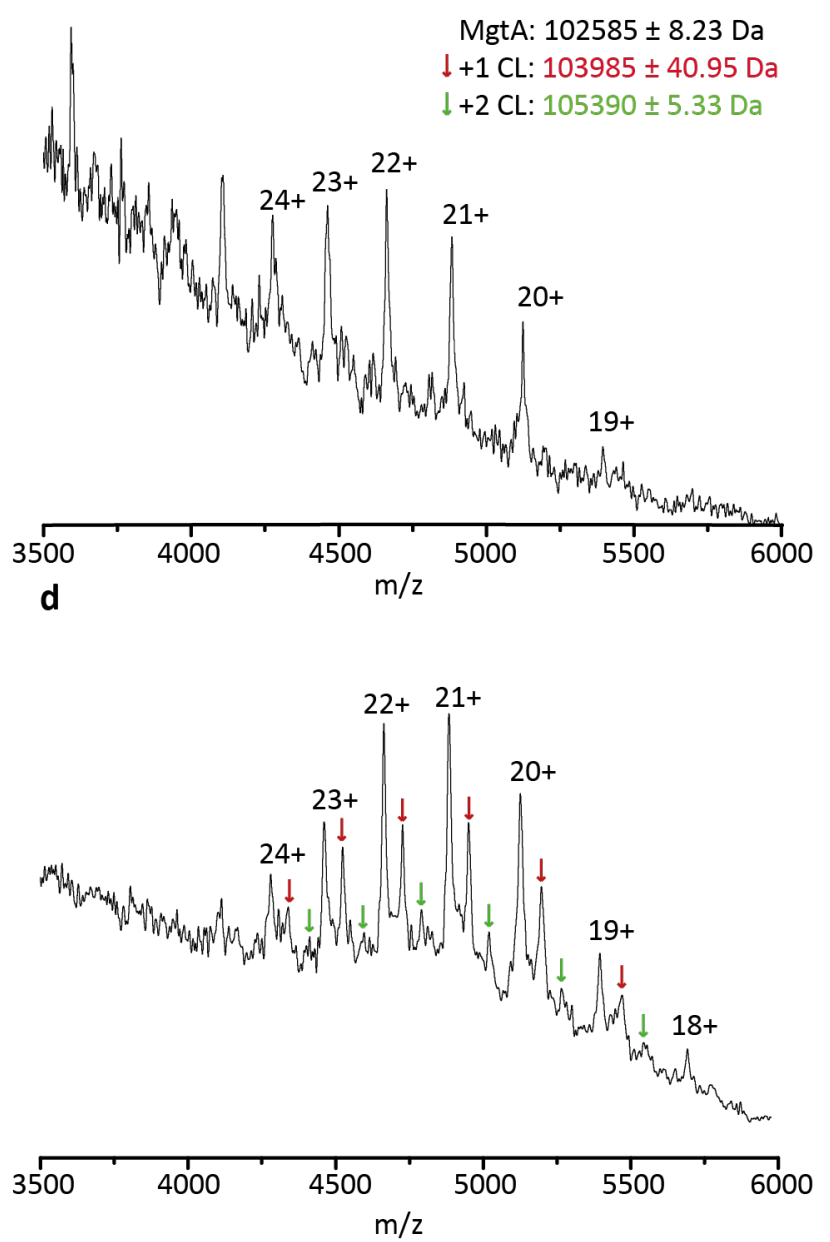

Supplemental figure 1: MgtA shows selective binding of cardiolipin in native mass spectrometry

Purified MgtA, solubilized in 3x CMC DDM, was analysed by native MS (a, b) in the absence or (c, d) presence of total $E$. coli lipid extract. Total $E$. coli lipid extract contains only $9.8 \%$ CL, while the remaining lipids are 57.5 \% PE, 15.1 \% PG and $17.6 \%$ unknown (Avanti Polar Lipids). The upper spectrum shows delipidated MgtA in DDM (19+ to 24+) with DDM clusters in the lower m/z region, while the lower spectra exhibits MgtA with E. coli lipid mixture (18+ to 24+) with a mixture of DDM clusters and lipids in the lower $\mathrm{m} / \mathrm{z}$ region. Close-up on MgtA spectra (right) reveals additions of approximately 1400 and $2800 \mathrm{Da}$ (red and green arrows) which correspond to the molecular weight of one and two cardiolipin molecules, respectively. The spectra were recorded in TOF mode and the key MS settings used to record these spectra are the voltages (sampling cone, trap collision, transfer collision and trap bias) and pressures (backing 
pressure, trap cell pressure). These values were $200 \mathrm{~V}, 150 \mathrm{~V}, 150 \mathrm{~V}, 45 \mathrm{~V}, 6.41$ mbar and $2.00 \times 10^{-2}$ mbar respectively.
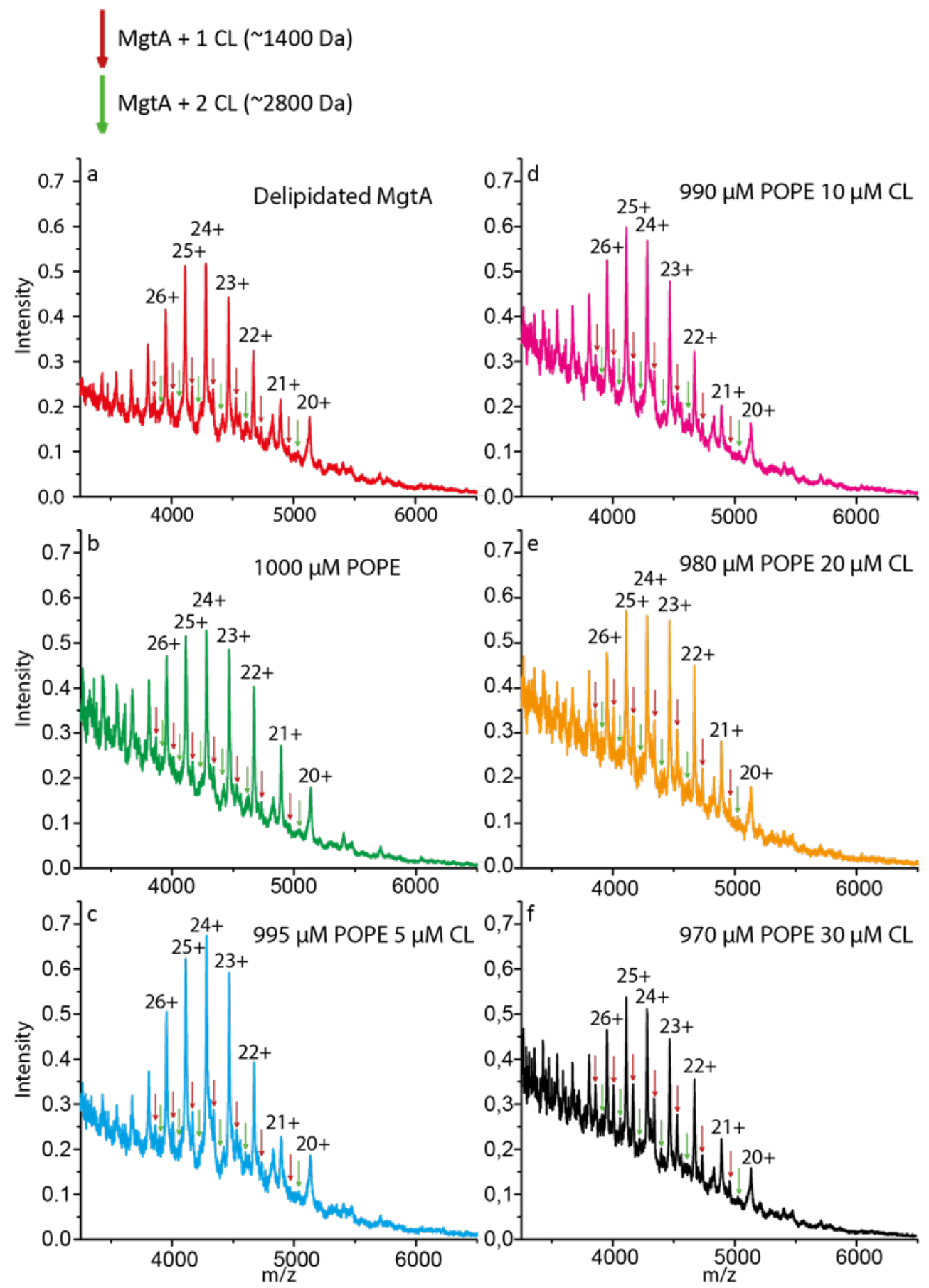

Supplemental figure 2: MS spectra showing a competition assay of MgtA with POPE and increasing CL 951 concentrations measured under activating conditions.

The concentrations in the spectra a to $\mathbf{f}$ of CL used is 0 to $30 \mu \mathrm{M} \mathrm{Cl}$ and 1000 to $970 \mu \mathrm{M}$ POPE lipid. The red arrow is the $+1 \mathrm{CL}(\sim 1400 \mathrm{Da})$ and the green arrow $+2 \mathrm{CL}(\sim 2800 \mathrm{Da})$. The spectra were recorded in TOF mode and the key MS settings are the voltages: sampling cone, trap collision, transfer collision, trap 
bias and pressures: backing pressure, trap cell pressure. These values where $150 \mathrm{~V}$,

A

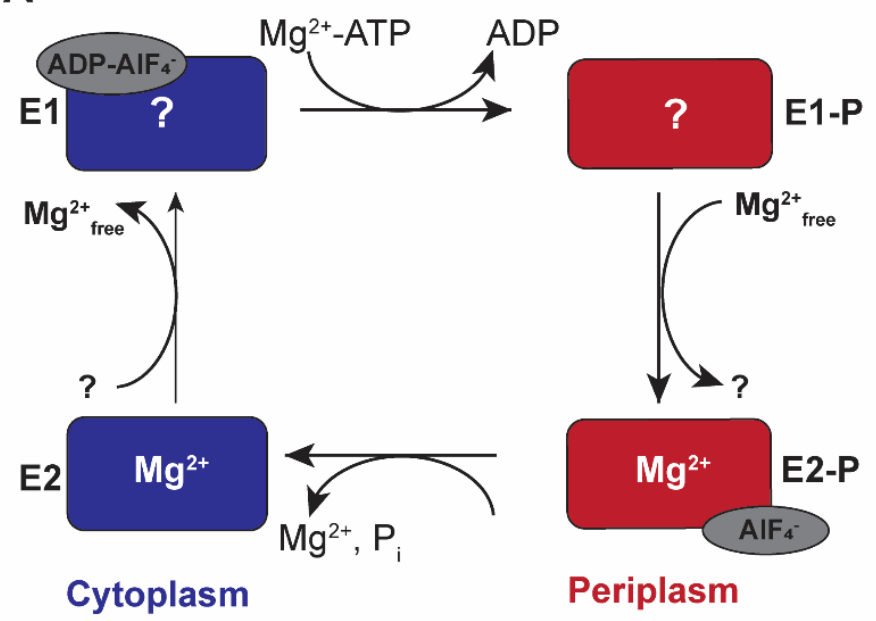

B

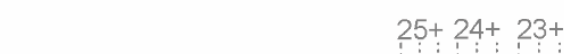

MgtA + lipid + AIF
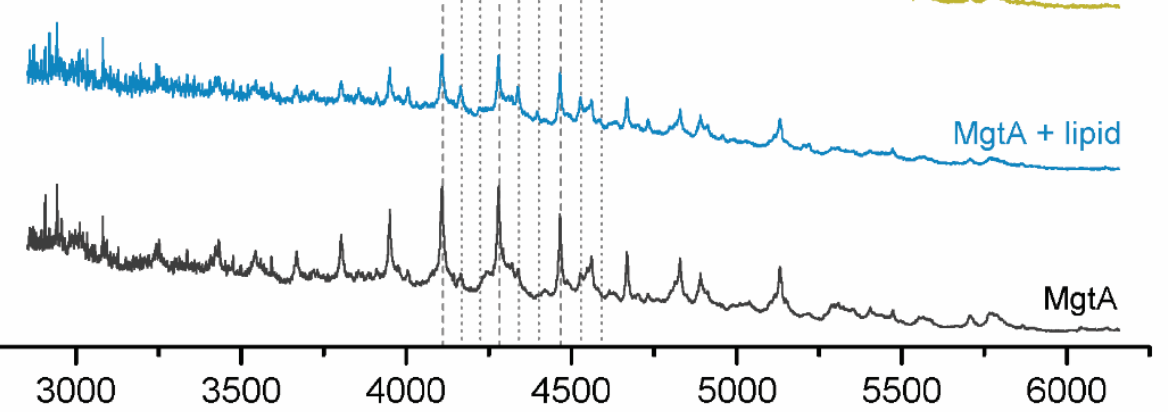

$\mathrm{m} / \mathrm{z}$

\section{Supplementary figure 3: Cardiolipin binding in the presence of MgtA inhibitors}

960 To determine binding of cardiolipin to MgtA dependent on the stages of transport cycle, lipid binding of

961 MgtA was determined in the presence of $\mathrm{AlF}_{4}^{-}$and $\mathrm{ADP}_{-} \mathrm{AlF}_{4}^{-}$, which lock MgtA in the E2 and E1 state,

962 respectively. 
(A) $\mathrm{Mg}^{2+}$-transport cycle of by MgtA is described by the Post-Albers cycle, in which MgtA alternates between different conformational states, termed E1 and E2 state. The E1 state has high affinity and is open for ions binding from the cytoplasm. The ion transported from the cytoplasm to the periplasm in the case of MgtA remains unknown. Upon ion binding, autophosphorylation of MgtA by ATP hydrolysis is induced, forming the E1P state. The phosphorylation induces domain rearrangements leading to the E2P state, which is now open to the periplasmic side. E2P has low affinity for the bound ions and high affinity for the counter ion, $\mathrm{Mg}^{2+}$. The exchange of counter ions dephosphorylates the enzyme and forms the E2 state. Upon further conformational changes the enzyme returns to the E1 state and the counterions are released into the cytoplasm. MgtA inhibitors, $\mathrm{AlF}_{4}^{-}$and $\mathrm{ADP}-\mathrm{AlF}_{4}^{-}$, lock the enzyme in the indicated conformational steps. (B) MgtA was incubated with inhibitors in 1:100 molar ratio for two hours, following incubation with total

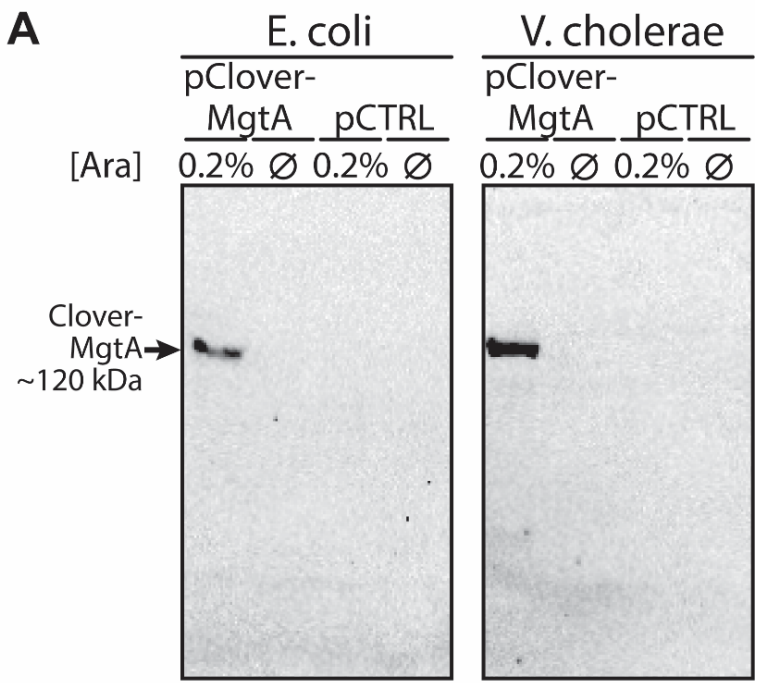

B

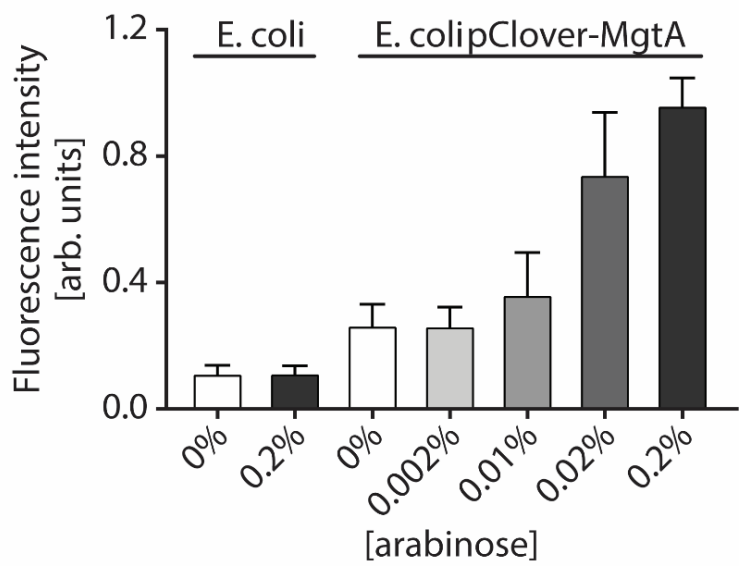




\section{Supplementary figure 4: Expression of Clover-MgtA}

981

982

983

984

985

986

987

988

989

990

991
(A) Western blot analysis of E. coli MG1655 and V. cholerae C6706 expressing Clover-fusion to MgtA. $E$. coli and $V$. cholerae containing either a plasmid encoding a translational fusion of Clover-MgtA under control of the arabinose inducible BAD promoter (pClover-MgtA) or the empty pBAD33.1 plasmid (pCTRL) were grown in the presence $(0.2 \%)$ or absence $(\varnothing)$ of arabinose. Samples of these cultures were analyzed by Western blot with a monoclonal antibody directed against GFP. (B) Quantification of CloverMgtA fluorescence by flow cytometry. E. coli containing the MgtA-Clover fusion plasmid (pClover-MgtA) or no plasmid were induced with different arabinose concentrations. The fluorescence intensity was quantified after excitation at $488 \mathrm{~nm}$. The bars depict the mean fluorescence, the error bars the standard deviation of at least 450,000 cells. 